\title{
A Finite-Time Thermodynamics of Unsteady Fluid Flows
}

\author{
Bernd R. Noack ${ }^{1, \star}$, Michael Schlegel ${ }^{1}$, Boye Ahlborn ${ }^{2}$, Gerd \\ Mutschke $^{3}$, Marek Morzyński ${ }^{4}$, Pierre Comte ${ }^{5}$ and Gilead Tadmor ${ }^{6}$ \\ ${ }^{1}$ Berlin Institute of Technology, Department of Fluid Dynamics and \\ Engineering Acoustics, Berlin, Germany \\ ${ }^{2}$ University of British Columbia, Department of Physics and Astronomy, \\ Vancouver, Canada \\ ${ }^{3}$ Forschungszentrum Dresden-Rossendorf, Institute of Safety Research, \\ Magnetohydrodynamics Department, Dresden, Germany \\ ${ }^{4}$ Poznań University of Technology, Institute of Combustion Engines and \\ Transportation, Poznań, Poland \\ ${ }^{5}$ Laboratoire d'Etudes Aérodynamiques (CNRS UMR 6609 / Université de \\ Poitiers), Poitiers, France \\ ${ }^{6}$ Northeastern University, Department of Electrical and Computer \\ Engineering, Boston, MA, USA
}

\section{^Corresponding author (BERND.R.NOACK@TU-BERLIN.DE)}

Communicated by K.H. Hoffmann, Chemnitz, Germany, and E. Michaelides, Denton, USA

\begin{abstract}
Turbulent fluid has often been conceptualized as a transient thermodynamic phase. Here, a finite-time thermodynamics (FTT) formalism is proposed to compute mean flow and fluctuation levels of unsteady incompressible flows. The proposed formalism builds upon the Galerkin model framework, which simplifies a continuum 3D fluid motion into a finite-dimensional phase-space dynamics and, subsequently, into a thermodynamics energy problem. The Galerkin model consists of a velocity field expansion in terms of flow configuration dependent modes and of a dynamical system describing the temporal evolution of the mode coefficients. Each mode is treated as one thermodynamic degree of freedom, characterized by an energy level. The dynamical system approaches local thermal equilibrium (LTE) where each mode has the same energy if it is governed only by internal (triadic) mode interactions. However, in the generic case of unsteady flows, the full system approaches only partial LTE with unequal energy levels due to strongly mode-dependent external interactions. The FTT model is
\end{abstract}


first illustrated by a traveling wave governed by a 1D Burgers equation. It is then applied to two flow benchmarks: the relatively simple laminar vortex shedding, which is dominated by two eigenmodes, and the homogeneous shear turbulence, which has been modeled with 1459 modes.

\section{Introduction}

A uniform flow encountering an obstacle breaks up into more or less random, laminar or turbulent structures, characterized as vortices or eddies. This vortex-shedding process is abundantly observed in nature and technology [1], for instance behind a bridge pier in a river (see Leonardo da Vinci's drawings [2]) and the flow around a mountain [3]. The eddies interact and gradually disappear in the wake of the obstacle. These structures acquire time-varying velocity components in the transverse and longitudinal directions out of the longitudinal flow. One can think of them either as interacting eddies (particles) or as time-varying streamwise structures (waves). The energy contained in these coherent structures is part of the internal energy of the fluid and, hence, is part of the thermodynamics of the system. Similar observations hold for many other shear flows as well, for instance jets or two merging streams with different velocities. In this study, a thermodynamic framework is proposed for such unsteady shear flows.

The phenomenology of shear flow depends on the Reynolds number, Re. At low values, the flow is steady. With increasing Reynolds numbers, instabilities occur and shape the coherent structures. Often, the first instability qualitatively represents the coherent structures observed far into the turbulent regime. Examples are the von Kármán vortex street behind a cylinder, the KelvinHelmholtz vortices in a shear layer, and the vortex rings in the Taylor-Couette configuration. The very ansatz of stability analysis rests on a steady base flow and fluctuations that are expanded in terms of space-dependent eigenmodes and time-dependent Fourier coefficients. Weakly nonlinear stability theory [4] accounts for nonlinear effects and thus extends the validity of the expansion ansatz. More generally, reduced-order Galerkin models may characterize not only the instability but also the irregular coherent structure dynamics [5]. Such models are generally based on modes arising from the spectral ansatz [6, 7], from stability analysis $[8,9]$, from empirical decompositions [5], or from physical insight $[10,11]$. These modes are global, i.e., extend over the entire flow domain - in contrast to finite elements and other computational bases. The beauty of these methods lies in the reduction of a complex space- and timedependent momentum transfer into a low-dimensional phase-space dynamics, where each direction is associated with a modal energy.

J. Non-Equilib. Thermodyn. 2008 · Vol. $33 \cdot$ No. 2 
The number of modes needed to resolve a turbulent flow is estimated by $N \approx R e^{9 / 4}$ [12]. Evidently, large Reynolds numbers imply that most degrees of freedom cannot be dynamically resolved but have to be statistically modeled. According to Boussinesq [13], the eddies act on the mean flow like an additional "eddy" viscosity $v_{\text {turb }}$. More generally, the effect of neglected finescale fluctuations on the dynamically resolved flow is represented by an additional eddy viscosity. This viscosity is estimated in Prandtl's mixing-length theory for turbulent flow [14]. Turbulent diffusion is depicted as an ensemble of eddies with velocity $u_{\text {mix }}$ and mean free path length $\lambda_{\text {mix }}$, called mixing length. That eddy viscosity is estimated by the product of the eddy velocity and its mixing length, $v_{\text {turb }} \sim \lambda_{\text {mix }} u_{\text {mix }}$, i.e., in complete analogy to kinetic theory for molecular viscosity $v \approx \lambda \bar{c}$, which depends on the average thermal speed $\bar{c}$ and the molecular mean free path $\lambda$. The success of the mixing-length theory comes from the fact that the new free parameters, the eddy velocity and mixing length, may be estimated algebraically much easier than the eddy viscosity itself. This mixing-length theory constitutes the core of most engineering turbulence simulations to date - including Reynolds-averaged Navier-Stokes (RANS) computations and large-eddy simulations (LES). Closer to the focus of this paper, reduced-order Galerkin models also rely on energy sinks to account for energy flow into unresolved structures [15-17].

The mixing-length theory provides a rather coarse statistical picture of the turbulent flow. In particular, it provides no account of the creation, decay, and interaction dynamics of these coherent structures. No systematic analogies have been established to date for such powerful thermodynamics concepts as degree of freedom, Boltzmann factor, equipartition of energy, entropy, entropy production (transfer of energy into the thermal background), and the principle of minimum entropy production. Numerous such analogies have been proposed on phenomenological grounds $[18,19]$ following the introduction of eddy viscosity. In particular, eddies have been suggested as "atoms" of turbulence. For grid turbulence, the energy of eddies has been experimentally observed to follow Maxwell-Boltzmann statistics [20]. In similar spirit, Chorin [21] points out a number of qualitative analogies between vortex motion and statistical physics. The statistical isotropic approximation of large-scale eddy motion is, however, inhibited for inhomogeneous shear flows due to their concentration in thin high-shear regions or their confinement by walls. Examples are wakes, jets, shear layers, and pipe flow.

In the "wave picture", particularly instructive are truncated Euler solutions [22] of a flow in a periodic box with vanishing base flow and neglecting viscous forces. In a spectral ansatz, this flow is approximated by a Galerkin expansion with a finite number of spatial Fourier modes and associated time-dependent amplitudes. The dynamical system for the mode amplitudes is derived from 
the Euler equation. The resulting solutions display equipartition of energy in all modes. While these solutions do not describe any real flow condition, this result suggests that the modes of a Galerkin approximation may nevertheless constitute a set of thermodynamic degrees of freedom to which equipartition of energy applies.

Here, we follow that hint. Targeting complex geometries, the restriction to a spectral method with Fourier modes [6] is relaxed to a traditional Galerkin model [23] with global orthonormal modes. This generalization allows the development of more efficient expansions for shear flows. While the spatial Fourier modes of the periodic box configuration represent sinusoidal waves in all directions, most modes of low-order Galerkin approximations of shear flows represent waves with a characteristic wavenumber and frequency, aligned with the convection velocity. These Galerkin models of incompressible flows usually do not obey equipartition of energy in the mode ensemble, since distinct modes are affected differently by the mean flow and viscous dissipation. Galerkin methods allow the quantification of how largescale energetic modes feed small-scale, less energetic ones via an energy flow cascade [24-26]. The energy absorbed by the large scales from the mean flow needs a finite time to be transferred to the small scales where it is dissipated. This energy transfer is reminiscent of finite-time thermodynamics [27-30] where the production and dissipation time scales are explicitly modeled. Differences between these time scales disturb equipartition of energy between modes. Our goal is to cast this "wave picture" in a systematic mathematical framework, framing a statistical physics analogy of the dynamics of coherent structures. We thus contribute to the link between statistical physics and fluid dynamics of unsteady fluid flows.

The paper is organized as follows. A finite-time thermodynamics (FTT) framework is outlined in Section 2, based on a Galerkin model. In Section 3, we discuss this FTT formalism with respect to nonlinear dynamics, control design, and thermodynamic analogies. In Section 4, the traveling wave solution of the 1D Burgers equation serves as a first illustration of the predictive capabilities of FTT models. Shear flow results are presented for the simple dynamics of 2D periodic vortex shedding (Section 5) and for the complex dynamics of the 3D homogeneous shear turbulence (Section 6). Finally, the main findings are summarized and open research problems are suggested in Section 7.

\section{Finite-time thermodynamics model}

Here, we propose a closure scheme for the computation of the first and second statistical moments of viscous incompressible fluid flows. Field dynamics

J. Non-Equilib. Thermodyn. 2008 · Vol. $33 \cdot$ No. 2 
are first transformed by the Galerkin method into a system of ordinary differential equations (Section 2.1). This dynamical system is characterized by ensemble-averaged equations for mean values and energy levels (Section 2.2). Employing the averaged equations, a finite-time thermodynamics consideration leads to a closure scheme (Section 2.3). Finally (Section 2.4), reduced systems are derived by lumping thermodynamic degrees of freedom.

\subsection{Galerkin model}

The incompressible flow is described in a Cartesian coordinate system $\mathbf{x}=$ $(x, y, z)$, where the $x$-axis is parallel to the flow, the $y$-axis is aligned with the shear, and the $z$-axis points in spanwise direction. The velocity field is expressed in the same coordinate system by $\mathbf{u}=(u, v, w)$. Time is denoted by $t$ and pressure by $p$. The fluid has kinematic viscosity $v$ and density $\rho$. In the following, all quantities are assumed to be nondimensionalized with the velocity scale $U$, the transverse length scale $D$, and the density $\rho$. The flow is characterized by the Reynolds number $\operatorname{Re}=U D / \nu$.

The flow domain $\Omega$ and the boundary conditions on the boundary $\partial \Omega$ are stationary. The employed boundary conditions have the form

$$
\mathcal{L}_{\mathrm{BC}}[\mathbf{u}]=\mathbf{F}_{\mathrm{BC}}(\mathbf{x}),
$$

where $\mathcal{L}_{\mathrm{BC}}$ is a linear operator and $\mathbf{F}_{\mathrm{BC}}(\mathbf{x})$ has a prescribed value. Examples are the homogeneous Dirichlet condition for no slip at a wall $\mathbf{u}=0$, the inhomogeneous variant for the oncoming velocity at infinity $\mathbf{u}=\mathbf{u}_{\infty}$, the Neumann condition with vanishing normal gradient $\partial_{n} \mathbf{u}=0$ as transverse boundary condition, a periodic assumption for infinite domains, and the convective outflow condition.

In FTT modeling, only the statistics of a converged flow solution is considered. These statistics is independent of the initial condition for the considered flows. Hence, the initial conditions need not be discussed. The conservation of mass, i.e., the equation of continuity, of an incompressible fluid reads

$$
\nabla \cdot \mathbf{u}=0
$$

The momentum (Navier-Stokes) equation is expressed by

$$
\mathcal{N}[\mathbf{u}]:=\partial_{t} \mathbf{u}+\nabla \cdot(\mathbf{u} \otimes \mathbf{u})+\nabla p-v \triangle \mathbf{u} \stackrel{!}{=} 0 .
$$

Here, $v:=1 / R e$. The pressure is not included as argument in the NavierStokes operator $\mathcal{N}$, since it is a function of $\mathbf{u}$. The pressure can be determined by the pressure Poisson equation derived from Eqs. (2) and (3). 
The flow field is decomposed into a steady, basic mode $\mathbf{u}_{0}$ and a time-varying fluctuation $\mathbf{u}^{\prime}$,

$$
\mathbf{u}(\mathbf{x}, t)=\mathbf{u}_{0}(\mathbf{x})+\mathbf{u}^{\prime}(\mathbf{x}, t),
$$

where both contributions satisfy the equation of continuity (2), $\nabla \cdot \mathbf{u}_{0}=\nabla$. $\mathbf{u}^{\prime}=0$, and the original and homogenized form of the boundary condition (1),

$$
\mathcal{L}_{\mathrm{BC}}\left[\mathbf{u}_{0}\right]=\mathbf{F}_{\mathrm{BC}}(\mathbf{x}) \quad \text { and } \quad \mathcal{L}_{\mathrm{BC}}\left[\mathbf{u}^{\prime}\right]=0 .
$$

The primary purpose of this decomposition is to apply the superposition principle to the fluctuation. Any linear combination of permissible fluctuations satisfies the equation of continuity and the boundary condition.

This opens the path for defining the Hilbert space $\mathcal{L}^{2}(\Omega)$ of square-integrable velocity fields for the fluctuation. The corresponding inner product is given by

$$
(\mathbf{u}, \mathbf{v})_{\Omega}:=\int_{\Omega} d \mathbf{x} \mathbf{u} \cdot \mathbf{v},
$$

and leads to the norm

$$
\|\mathbf{u}\|_{\Omega}:=\sqrt{(\mathbf{u}, \mathbf{u})_{\Omega}} .
$$

This Hilbert space has a complete, countable system $\left\{\mathbf{u}_{i}(\mathbf{x})\right\}_{i=1}^{\infty}$ as basis. Without loss of generality, orthonormality can be assumed,

$$
\left(\mathbf{u}_{i}, \mathbf{u}_{j}\right)_{\Omega}=\delta_{i j}, \quad i, j=1, \ldots, N .
$$

Thus, the flow (4) can be approximated by a Galerkin expansion $\mathbf{u}^{[0 \ldots N]}$ with $N$ space-dependent modes $\mathbf{u}_{i}$ and corresponding time-dependent Fourier coefficients $a_{i}$,

$$
\mathbf{u}(\mathbf{x}, t) \approx \mathbf{u}^{[0 \ldots N]}(\mathbf{x}, t):=\mathbf{u}_{0}(\mathbf{x})+\sum_{i=1}^{N} a_{i}(t) \mathbf{u}_{i}(\mathbf{x}),
$$

where $a_{i}=\left(\mathbf{u}^{\prime}, \mathbf{u}_{i}\right)_{\Omega}, i=1, \ldots, N$.

The evolution equation for the Fourier coefficients is derived from the NavierStokes equation via a Galerkin projection onto each mode,

$$
\left(\mathbf{u}_{i}, \mathcal{N}\left[\mathbf{u}^{[0 \ldots N]}\right]\right)_{\Omega}=0, \quad i=1, \ldots, N .
$$

The resulting Galerkin system contains constant, linear, and quadratic terms in $a_{i}, i=1, \ldots, N$ : 


$$
\dot{a}_{i}=c_{i}+\sum_{j=1}^{N} l_{i j} a_{j}+\sum_{j, k=1}^{N} q_{i j k} a_{j} a_{k}, \quad i=1, \ldots, N .
$$

The coefficients $c_{i}, l_{i j}$ and $q_{i j k}$ are derived in [26].

In principle, almost any incompressible flow can be arbitrarily accurately described by the "traditional" Galerkin method, as described above (see, e.g., [23]). Hence, the assumption of an existing model poses no severe limitation on the applicability of the FTT formalism. In practice, the computational cost of this $O\left(N^{3}\right)$ algorithm requires that $N$ be small. Exceptions to this rule may be spectral methods with an $N \log N \operatorname{cost}[6,7]$.

The art of efficient Galerkin modeling rests largely on designing the modes for particular configurations. The literature suggests mathematical [31], physical [9], and empirical approaches [5] serving different goals. In the mathematical approach, completeness of the Galerkin expansion is guaranteed. In fact, mathematical Galerkin methods are used to prove properties of the NavierStokes solution [32]. The physical method utilizes stability eigenmodes, thus economically describing linear behavior. In the empirical direction, e.g., using the Proper Orthogonal Decomposition (POD), nonlinear dynamics may be optimally resolved in a certain sense by extracting the most energetic structures from a known flow solution. The current study employs a mathematical expansion for the Burgers equation (Section 4), an empirical model for the wake flow (Section 5), and a physical one for the shear turbulence (Section 6).

\subsection{Ensemble-averaged equations}

The proposed FTT formalism rests on the traditional Galerkin model and shall predict only ensemble means. For the transient onset of an instability (Appendix B) or the decay of turbulence (Appendix C), such a filter may be approximated by a phase or short-term average. For post-transient behavior (Sections 4-6), statistical stationarity and ergodicity are assumed, i.e., the ensemble average is equivalent to the time average, and time derivatives of ensemble-filtered quantities vanish.

In the following, ensemble-averaged quantities are denoted by an overbar and the corresponding fluctuation by a prime, e.g., $a_{i}=\overline{a_{i}}+a^{\prime}$. The ensembleaveraged Galerkin system (6) reads

$$
\dot{\overline{a_{i}}}=c_{i}+\sum_{j=1}^{N} l_{i j} \overline{a_{j}}+\sum_{j, k=1}^{N} q_{i j k} \overline{a_{j}} \overline{a_{k}}+\sum_{j, k=1}^{N} q_{i j k} \overline{a_{j}^{\prime} a_{k}^{\prime}}, \quad i=1, \ldots, N .
$$


This equation shall be called Galerkin-Reynolds equation as it can also be derived by a Galerkin projection onto the ensemble-averaged momentum (Reynolds) equation,

$$
\left(\mathbf{u}_{i}, \overline{\mathcal{N}\left[\mathbf{u}^{[0 \ldots N]}\right]}\right)_{\Omega}=0, \quad i=1, \ldots, N .
$$

The evolution equation of the modal energy $E_{i}:=\overline{\left(a_{i}^{\prime}\right)^{2}} / 2$ is derived by multiplying Eq. (6) with $a_{i}^{\prime}$ and averaging,

$$
\dot{E}_{i}=Q_{i}+T_{i}, \quad i=1, \ldots, N,
$$

where

$$
\begin{aligned}
Q_{i} & :=\sum_{j=1}^{N} q_{i j} \overline{a_{i}^{\prime} a_{j}^{\prime}}, \quad q_{i j}:=l_{i j}+\sum_{k=1}^{N}\left(q_{i j k}+q_{i k j}\right) \overline{a_{k}}, \\
T_{i} & :=\sum_{j, k=1}^{N} T_{i j k}, \quad T_{i j k}:=q_{i j k} \overline{a_{i}^{\prime} a_{j}^{\prime} a_{k}^{\prime}} .
\end{aligned}
$$

A detailed analysis [26] reveals that the first term $Q_{i}$ originates from NavierStokes terms that are linear with respect to $\mathbf{u}^{\prime}$ comprising modal production, convection, and dissipation terms. These linear terms represent external interactions with the mean flow $\overline{\mathbf{u}}$ as a "macroscopic background" or the molecular chaos via viscosity as a "microscopic background". The second term $T_{i}$ originates from the quadratic convection term in Eq. (3) and is known as the transfer term. This term comprises intermodal energy exchange rates. The effect of the pressure term in this modal energy flow balance either vanishes exactly for closed flows [5], approximately for open flows in large domains [25,33], or adds to $Q_{i}$ and $T_{i}$ in analogy to the velocity fluctuation. The effect of pressure power on $Q_{i}$ results from interactions with mean flow and on $T_{i}$ from intermodal interactions.

\subsection{Closure model}

Equations (7) and (8) represent $2 N$ equations for $2 N$ unknowns, the mean values and energy levels of each mode: $\left\{\bar{a}_{i}\right\}_{i=1}^{N},\left\{E_{i}\right\}_{i=1}^{N}$. However, the equations contain unknown second- and third-order centered statistical moments, $\overline{a_{i}^{\prime} a_{j}^{\prime}}$ and $\overline{a_{i}^{\prime} a_{j}^{\prime} a_{k}^{\prime}}$. Equations for these moments can be derived from Eq. (6) but at the price of generating more unknown higher-order statistical moments. The resulting system of equation can never be closed, constituting the well-known closure problem of turbulence. In this section, we introduce closure approximations for these moments. In particular, we aim to parameterize all centered moments by the modal energy distribution. 
The external interaction term $Q_{i}$ requires an ansatz for the second centered moments of the Fourier coefficients. We assume a diagonal correlation matrix,

$$
\overline{a_{i}^{\prime} a_{j}^{\prime}}=2 E_{i} \delta_{i j}, \quad i, j=1, \ldots, N .
$$

This assumption is fulfilled by definition for the Proper Orthogonal Decomposition (POD) - also called the Karhunen-Ldeve decomposition or Principal Components Analysis (PCA). It is approximately fulfilled also by locally timeperiodic shear flows resolved by Fourier modes [34], POD modes [24,26,33], or stability eigenmodes of oscillatory nature [25]. Here, each harmonic frequency is resolved by one pair of modes mutually shifted by a quarter period with similar pair-wise energy content. The analysis of this paper remains valid for non-POD modes or non-periodic flows when deviations from Eq. (9) by individual $(i, j)$ pair cancel out approximately in the summation of formula (8) for $Q_{i}$. The ultimate corroboration in such cases is by numerical experiments, as provided below.

Equation (9) simplifies the Galerkin-Reynolds equation to

$$
\dot{\overline{a_{i}}}=c_{i}+\sum_{j=1}^{N} l_{i j} \overline{a_{j}}+\sum_{j, k=1}^{N} q_{i j k} \overline{a_{j}} \overline{a_{k}}+\sum_{j=1}^{N} 2 q_{i j j} E_{j}, \quad i=1, \ldots, N .
$$

The energy distribution $\left\{E_{i}\right\}_{i=1}^{N}$ parameterizes the mean values $\left\{\overline{a_{i}}\right\}_{i=1}^{N}$. The simplified Galerkin-Reynolds equation has a number of intriguing limiting cases. Let $\mathbf{u}_{0}$ represent the steady Navier-Stokes solution $\mathbf{u}_{s}$. Then $c_{i}=0$, $i=1, \ldots, N$, and, by a first-order approximation, the mean values depend linearly on the energy distribution - in agreement with mean-field theory [4]. If $\mathbf{u}_{0}=\overline{\mathbf{u}}$ is chosen to be the mean solution of a statistically stationary dynamics, $\overline{a_{i}}=0, i=1, \ldots, N$ and Eq. (10) represents a linear system from which the energy distribution can be determined. When $\mathbf{u}_{0}$ is not too far from the mean solution, then quadratic terms in $\overline{a_{i}}$ can be neglected and the changes of the averaged flow depend linearly on the energy distribution.

The assumption (9) implies that the modal external interaction term is only a function of the corresponding modal energy,

$$
\hat{Q}_{i}=\hat{q}_{i} E_{i}, \quad \text { where } \quad \hat{q}_{i}:=2 q_{i i}+2 \sum_{j=1}^{N}\left(q_{i i j}+q_{i j i}\right) \overline{a_{j}}, \quad i=1, \ldots, N .
$$

Here and throughout, a hat shall indicate the (approximate) FTT-modeled quantity as opposed to its exact representation from the Navier-Stokes equation. 
The transfer or internal interaction term $T_{i}$ is the sum of many triadic interaction terms $T_{i j k}$. The energy exchanges in each triad conserves the total energy $E_{i}+E_{j}+E_{k}$, due to the energy-preserving (Hamiltonian) property of the quadratic Galerkin system term (see Appendix A). In other words, the total energy of a triad is only redistributed between the modes. We assume that the triadic interaction term is a function of the modal energies of the involved modes, $\hat{T}_{i j k}=\hat{T}_{i j k}\left(E_{i}, E_{j}, E_{k}\right)$ and that energy flows downhill, i.e., from more to less energetic modes. With this ansatz, a mathematical procedure (see Appendix A) leads to

$$
\begin{gathered}
\hat{T}_{i j k}=\alpha \chi_{i j k}\left(E_{i} E_{j} E_{k}\right)^{1 / 2}\left(\frac{\frac{1}{2}\left(E_{j}+E_{k}\right)-E_{i}}{E_{i}+E_{j}+E_{k}}\right), \\
i, j, k=1, \ldots, N,
\end{gathered}
$$

where $\alpha$ is derived from a condition of energy flow consistency (A 8) and the triadic interaction function

$$
\chi_{i j k}:=\frac{1}{6}\left(\left|q_{i j k}\right|+\left|q_{i k j}\right|+\left|q_{j i k}\right|+\left|q_{j k i}\right|+\left|q_{k i j}\right|+\left|q_{k j i}\right|\right)
$$

is determined by the Galerkin system.

The resulting FTT-modeled energy equation reads

$$
\dot{E}_{i}=\hat{q}_{i} E_{i}+\sum_{j, k=1}^{N} \hat{T}_{i j k}\left(E_{i}, E_{j}, E_{k}\right), \quad i=1, \ldots, N
$$

Donor modes with $\hat{q}_{i}>0$ provide energy from the external background in the mode system while the recipient modes with $\hat{q}_{i}<0$ discharge this energy. The transfer term is energy preserving and redistributes energy toward a local thermal equilibrium $\left(E_{1}=E_{2}=\ldots=E_{N}\right)$. In highly energetic modes we find $\hat{q}_{i}>0$. The first term therefore promotes "capitalism" by making "rich" donor modes even richer, while the second term can be viewed as social welfare in the mode sociology.

Equations (10) and (13) constitute our FTT equations, representing $2 N$ nonlinear equations for $2 N$ statistical moments, $\left\{\bar{a}_{i}\right\}_{i=1}^{N}$ and $\left\{E_{i}\right\}_{i=1}^{N}$. These equations accommodate the steady solution $E_{1}=E_{2}=\ldots=E_{N}=0$ in addition to a potentially unsteady solution - assuming the Galerkin model to be accurate. Figure 1 illustrates the interdependency between averaged Fourier coefficients and modal energy distributions. 


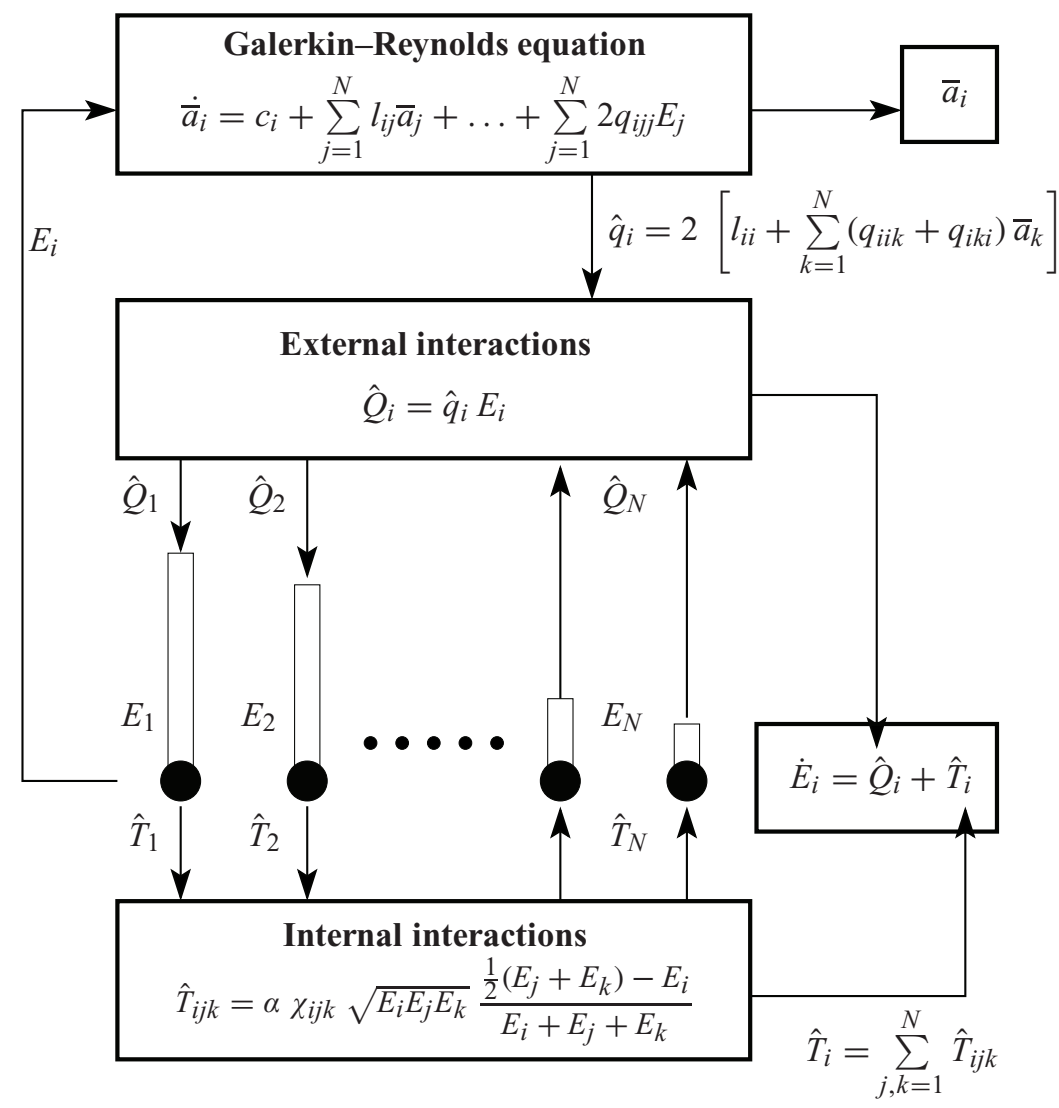

Figure 1 FTT model: Interrelation between the Galerkin-Reynolds equation (10) and the modal energy balance equation (13).

\subsection{System reduction}

Geometric symmetry conditions lead to equipartition of energy in certain groups of modes [35]. In particular, Galerkin models commonly employ pairs of modes to span distinct traveling waves with characteristic wavenumbers and frequencies [24-26]. The modes of such pairs have the same or very similar energies, which are periodically transferred back and forth between them. An analytical argument is provided in [36]. Let us assume that modes $i=1$ and $i=2$ form such a pair. This pairwise equipartition $E_{1}=E_{2}$ may be employed to reduce the order of the energy equation (13). The first two equations are lumped into one for $E^{[1,2]}:=E_{1}+E_{2}$,

$$
\dot{E}^{[1,2]}=\frac{\hat{q}_{1}+\hat{q}_{2}}{2} E^{[1,2]}+\sum_{j, k=1}^{N}\left(\hat{T}_{1 j k}+\hat{T}_{2 j k}\right) .
$$


While the external interaction term $\hat{Q}^{[1,2]}:=\hat{Q}_{1}+\hat{Q}_{2}$ can be expressed in terms of $E^{[1,2]}$, the internal interaction term uses $E_{1}=E_{2}=E^{[1,2]} / 2$ and further simplifications require conditions on the triadic interaction function $\chi_{i j k}$.

The considerations above may easily be generalized to more and larger subsets of modes living in equipartition of energy. A trivial, extreme case is where all modes are included in a single group $\mathcal{I}:=\{1, \ldots, N\}$. Energy conservation means that $\sum_{i, j, k=1}^{N} \hat{T}_{i j k}=0$. Hence, an equilibrium implies a zero net external interaction

$$
\dot{E}^{[1 \ldots N]}=\left[\left(\hat{q}_{1}+\ldots+\hat{q}_{N}\right) / N\right] E^{[1 \ldots N]}=0 .
$$

This further implies either the trivial steady solution

$$
E_{1}=\ldots=E_{N}=0
$$

or that external interaction coefficients satisfy $\hat{q}_{1}+\ldots+\hat{q}_{N}=0$.

A generic nontrivial "least-order" model may be based on the classification into donor modes with indices in $\mathcal{I}^{+}:=\left\{i \in \mathcal{I}: \hat{q}_{i}>0\right\}$ and recipient modes associated with $\mathcal{I}^{-}:=\left\{i \in \mathcal{I}: \hat{q}_{i} \leq 0\right\}$. This least-order lumping is employed to determine $\alpha$ in Eq. (12) (see Appendix A).

A particularly attractive feature of Eq. (13) is the possibility to compute parameters of simplified energy distributions. Let, for instance, $E_{i}=a+i b$ represent a linear approximation. Then, a lowest (nontrivial) minimum of the residual $\chi^{2}(a, b):=\sum_{i=1}^{N}\left(\hat{q}_{i} E_{i}+\sum_{j, k=1}^{N} \hat{T}_{i j k}\right)^{2}$ indicates a good choice of $a, b$.

\section{Nonlinear dynamics, control theory, and thermodynamics analogies}

The FTT model is discussed in this section from dynamical systems, control theory, and thermodynamics perspectives. First (Section 3.1), the dynamical behavior is classified. Nonlinear control opportunities are distilled in Section 3.2. Finally, mathematical analogies of the FTT system as heat conduction (Section 3.3) and spectroscopy problem (Section 3.4) are presented. 


\subsection{Classification of model behavior}

The solutions of the modal energy equations (13) depend on the external interaction efficiencies $\hat{q}_{i}$. For the sake of simplicity, we assume here that these parameters are constant, i.e., neglect the interdependency between $\left\{\hat{q}_{i}\right\}_{i=1}^{N}$ and the energy distribution $\left\{E_{i}\right\}_{i=1, \ldots ., N}$ communicated by the mean values $\left\{\bar{a}_{i}\right\}_{i=1}^{N}$. Furthermore, we assume that all modes are connected via the internal triadic interaction terms $\hat{T}_{i j k}$ and that there are "no isolated islands of modes". Precisely, two modes $i$ and $j$ are said to be directly connected if there exists a $k$ such that $\chi_{i j k} \neq 0$. They are indirectly connected if they are not directly connected but there exists a chain of $K$ modes, $l_{1}, l_{2}, \ldots, l_{K}$ such that the pairs $\left(i, l_{1}\right),\left(l_{1}, l_{2}\right), \ldots,\left(l_{K}, j\right)$ are directly connected. The minimum value of $K$ defines the order of connection. In these terms we assume that any pair of modes is either directly or indirectly connected, guaranteeing energy transfer coupling between all modes.

Under these assumptions, four kinds of FTT model behavior (13) can be classified:

A) Hamiltonian dynamics $\left(\min _{i \in \mathcal{I}} \hat{q}_{i}=\max _{i \in \mathcal{I}} \hat{q}_{i}=0\right)$ :

The total energy $E$ of the initial condition is preserved and the system can be expected to approach a state with approximate equipartition of energy,

$$
\lim _{t \rightarrow \infty} E_{i}(t)=E / N, \quad i=1, \ldots, N
$$

In the social analogy of Section 2.3, social welfare between the modes leads to complete communism.

B) Dissipative dynamics $\left(\min _{i \in \mathcal{I}} \hat{q}_{i}<\max _{i \in \mathcal{I}} \hat{q}_{i} \leq 0\right)$ :

The system converges to the origin,

$$
\lim _{t \rightarrow \infty} E_{i}(t)=0, \quad i=1, \ldots, N .
$$

Social welfare continually provides the recipient modes with $\hat{q}_{i}<0$ with energy, until all energy is lost.

C) Anti-dissipative dynamics $\left(0 \leq \min _{i \in \mathcal{I}} \hat{q}_{i}<\max _{i \in \mathcal{I}} \hat{q}_{i}\right)$ :

The system converges to infinity,

$$
\lim _{t \rightarrow \infty} E_{i}(t)=\infty, \quad i=1, \ldots, N .
$$

This behavior corresponds to case B in reverse time direction. 
D) Hyperbolic dynamics $\left(\min _{i \in \mathcal{I}} \hat{q}_{i}<0<\max _{i \in \mathcal{I}} \hat{q}_{i}\right)$ :

On the basis of our numerical results, the system is conjectured to converge for all initial conditions with nonvanishing energies $E_{i}>0$, $i=1, \ldots, N$, to a unique stable flow equilibrium

$$
\lim _{t \rightarrow \infty} E_{i}(t)=E_{i}^{\infty}, \quad i=1, \ldots, N .
$$

Social welfare prevents the rich donor modes from divergence and the recipient modes from dying.

Under the premise of complete nonlinear connectivity, the four cases constitute a classification of dynamics behavior, i.e., any FTT model (13) belongs to one and only one of the cases. The conjectured nonlinear behavior for cases A and D is consistent with all the FTT studies carried out by the authors, including those presented here.

Case A and D are the only nontrivial bounded behaviors. The Hamiltonian dynamics A leads to local thermal equilibrium (LTE) in a micro-ensemble of modes that has no energy exchange with the outside world. The converged state of hyperbolic dynamics can be considered as a partial LTE of a macro-ensemble of modes that exchanges energy with the background. The background "heats" the donor modes and "cools" the recipient modes, thus preventing complete LTE. In general, large (small) $\hat{q}_{i}$ correlate with large (small) $E_{i}$. However, $\hat{q}_{i}>\hat{q}_{j}$ does not generally imply $E_{i}>E_{j}$, since the $\hat{T}_{i j k}$ redistributes energy at rates determined also by $\chi_{i j k}$. A "greedy" donor mode with a small internal exchange rate can build larger wealth than another donor mode that overcompensates a larger $\hat{q}_{i}$ by an overproportional contribution to the social welfare system.

The hyperbolic dynamics is a typical case for Galerkin models of unstable shear flows and is implicitly assumed in the following, unless otherwise stated. From our numerical examples, we conjecture that this dynamics has only one stable fixed point $\left(E_{1}^{\infty}, \ldots, E_{N}^{\infty}\right)$, i.e., it cannot display hysteresis with two or more stable states. However, multi-attractor behavior may arise if the assumption of frozen $\hat{q}_{i}$ is dropped. In this case, the energy distribution can change the $\hat{q}_{i}$ via the mean solution $\left\{\bar{a}_{i}\right\}_{i=1}^{N}$.

\subsection{Controlled dynamics}

The FTT model can be employed as a plant for infinite-horizon, nonlinear control design. A thorough discussion of flow control using this framework requires an independent presentation, covering modeling, actuator implementa- 
tion, dynamic estimation, and ways to address practical performance-limiting issues. These are currently being pursued by the authors. Here we shall be content with a brief sketch. It is focused on the ideal case of a single volume force actuator with a commanded, time-varying amplitude $b(t)$. This force enters the Galerkin system (6) in the form

$$
\dot{a}_{i}=c_{i}+\sum_{j=1}^{N} l_{i j} a_{j}+\sum_{j, k=1}^{N} q_{i j k} a_{j} a_{k}+g_{i} b, \quad i=1, \ldots, N,
$$

where $g_{i}$ are mode-dependent gains [37-39]. The ubiquitous acoustic actuator is an example [40].

With a linear state feedback law,

$$
b:=\sum_{j=1}^{N} k_{j} a_{j}
$$

the resulting controlled dynamics is described by

$$
\dot{a}_{i}=c_{i}+\sum_{j=1}^{N} l_{i j}^{c} a_{j}+\sum_{j, k=1}^{N} q_{i j k} a_{j} a_{k}, \quad i=1, \ldots, N,
$$

where $l_{i j}^{c}:=l_{i j}+g_{i} k_{j}$ and the quadratic term is not changed. Hence, the prerequisites of the FTT model with a Hamiltonian quadratic term are fulfilled by Eq. (16). Thus, the nonlinear dynamical effect of the control law (15) is predicted by the FTT model over an infinite time horizon, enabling a fully nonlinear, infinite-horizon control design.

Generic flow control objectives can be stated in terms of targeted modal energies. A common example is the suppression of fluctuations [41,42]. Excitation of selected structures as a means to enhance mixing is another. Control enters the energy flow equation via the added modal actuation power $G_{i}:=g_{i} \overline{a_{i}^{\prime} b}$ :

$$
\dot{E}_{i}=Q_{i}+T_{i}+G_{i}, \quad i=1, \ldots, N .
$$

In these terms, suppression of fluctuations is achieved by imposing dissipative dynamics. The dissipation can be imposed directly on the energy of the larger structures in donor modes $E^{+}:=\sum_{i \in \mathcal{I}^{+}} E_{i}$. If the donor modes die out and do not feed the recipient modes, the latter will die, too. In the linear feedback framework (15), this is achieved by selecting $k_{i}=2\left(\sigma^{+}-\hat{q}_{i}\right) / g_{i}, \quad i \in \mathcal{I}_{+}$, where $\sigma^{+}<0$ is the minimal desired decay rate. Indeed, by partitioning the original system into donors and recipient states, as done earlier, this formalism guarantees that the following closed loop system is dissipative: 


$$
\begin{aligned}
& \dot{E}^{+}=\sum_{i \in \mathcal{I}^{+}}\left(Q_{i}+T_{i}+G_{i}\right)=\sigma^{+} E^{+}+T^{+}, \\
& \dot{E}^{-} \leq \hat{q}^{-} E^{-}+T^{-},
\end{aligned}
$$

where $\hat{q}^{-}=\max _{i \in \mathcal{I}^{-}} \hat{q}_{i}<0$ and $T^{+}+T^{-}=0$. In analogy to $E^{+}$, the superscripts "+" ("-") denote aggregate terms for the donor (recipient) modes. Not surprisingly, this control law is equivalent to energy-based control variants that have successfully been employed to stabilize wake flows using a Galerkin model for control design [43].

An alternative approach to stabilization is the actual stimulation of growth in selected energy recipients. Although counterintuitive, at first glance, this kind of control builds on the fact that triadic energy exchange terms (12) depend on the amplitude of the consumer energy. An increase in the latter can increase the rate of energy transfer from donors to consumers, and thus decrease the overall energy of the system. This is the rationale behind the ubiquitous use of high frequency jet actuation to attenuate low frequency instabilities. Controller parameters can be chosen by optimization of Eq. (16). We leave out the details.

\subsection{Analogy with heat conduction}

For reasons of simplicity, the discussion of the triadic interaction model employs Eq. (A 10), from Appendix A, but could easily be generalized to Eq. (12). This model retransfers energy from the most energetic to the least energetic modes. The implied behavior of the modal interactions terms $\hat{T}_{i j k}, \hat{T}_{j i k}$, and $\hat{T}_{k i j}$ from a triad at given energy levels $E_{i}, E_{j}$, and $E_{k}$ is geometrically visualized in Figure 2. The ansatz predicts vanishing transfer between all modes in case thermal equilibrium is achieved, $E_{i}=E_{j}=E_{k}$. Thus, the modeled energy transfer between modes qualitatively resembles Fick's law, describing the energy transfer by diffusion across temperature gradients. Here, the flux ceases when equilibrium distribution is reached. Hence, we propose to call the triadic interaction model Fick's law for triadic interactions.

That analogy with heat fluxes can be made more concrete in a mechanical analogy. Each mode at energy $E_{i}$ (solid circles in Figure 2) may be identified with a sphere with unit heat capacity and temperature $\theta_{i}$. In addition, auxiliary spheres are introduced corresponding to the averaged energy between two modes (open circles in Figure 2) and containing negligible heat capacity. The auxiliary sphere connecting the $j$-th and $k$-th element can be maintained at the average temperature $\theta_{j k}:=\left(\theta_{j}+\theta_{k}\right) / 2$ by the same heat conductor to both elements. Equation (A 10) can now be interpreted as heat conduction 


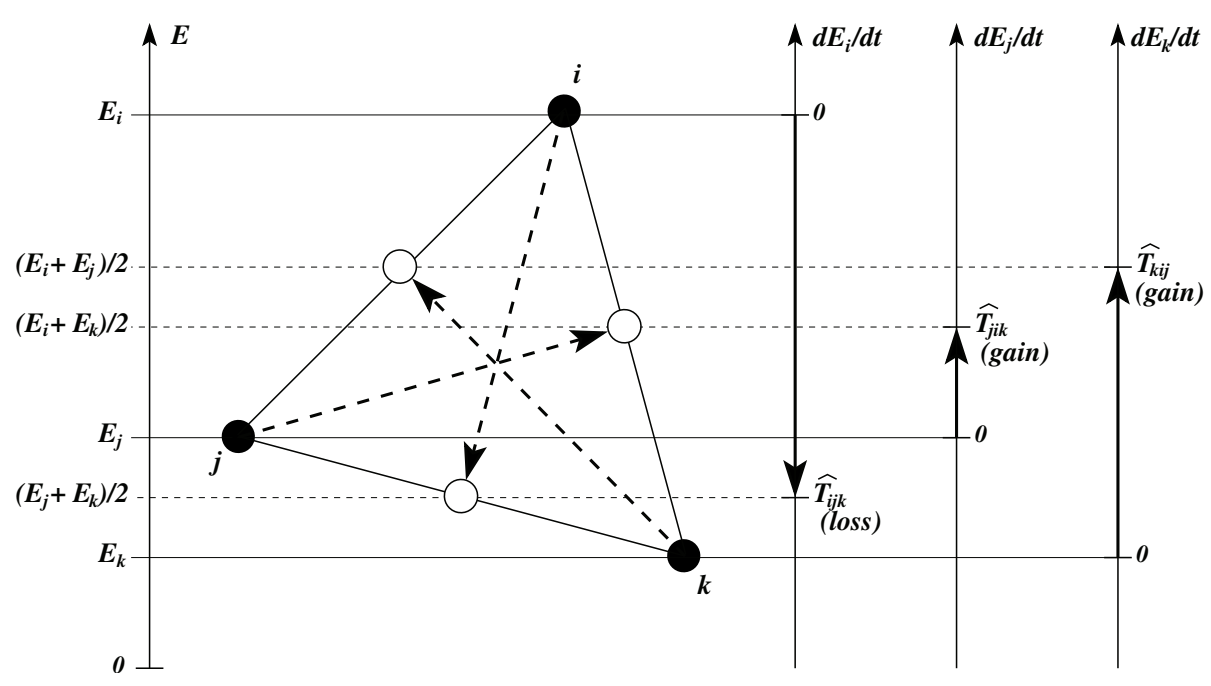

Figure 2 Geometric interpretation of Fick's law for triadic interactions. Each vertex (solid circle) of the isometric triangle represents one mode $i, j$, or $k$. Without loss of generality, the triangle is arranged so that the horizontal projection of the vertices denotes $E_{i}, E_{j}$, and $E_{k}$, respectively (left axis). Similarly, the midpoints (open circles) represent averaged energy levels of two modes. Formula (A 10) implies that the modeled energy exchange rates $\hat{T}_{i j k}, \hat{T}_{j i k}$, and $\hat{T}_{k i j}$ are proportional to the horizontal projection of the bisectors associated with mode $i, j$, and $k$, respectively (right arrows). The energy flow toward one mode is positive (negative) if the averaged energy of the remaining modes is larger (smaller), i.e., energy flows "downhill".

between the $i$-th sphere and the corresponding auxiliary sphere in that triad. The heat flux depends on the temperature of all three spheres. The ensemble of modes represents an ensemble of spheres (including the auxiliary ones). The set of triadic interactions are reflected in the conductor network. Positive (negative) external energy flows $Q_{i}$ correspond to external heating (cooling) of the $i$-th sphere. This completes the analogy of the modal energy balance equation (13).

\subsection{Analogy with spectroscopy}

Another thermodynamic analogy can be established with the Einstein transition probabilities in spectroscopy [44]. Fick's law of triadic interactions can be interpreted as two bi-modal interactions, writing Eq. (A 10) as

$$
\begin{aligned}
\hat{T}_{i j k}= & \alpha\left(E_{i} E_{j} E_{k}\right)^{1 / 6}\left[\frac{1}{2}\left(E_{j}-E_{i}\right)+\frac{1}{2}\left(E_{k}-E_{i}\right)\right], \\
& i, j, k=1, \ldots, N .
\end{aligned}
$$


In the framework of the analogy, this corresponds to two simultaneous transitions between three energy levels. Obviously, mode $i$ takes energy from modes $j$ and $k$ if their levels are higher and gives energy if their levels are lower.

This bi-modal interpretation leads to a transition matrix formalism,

$$
\begin{aligned}
& \hat{T}_{i}=\sum_{j, k=1}^{N} \hat{T}_{i j k}=\sum_{j=1}^{N} A_{i j} E_{j}, \quad i=1, \ldots, N, \\
& A_{i j}:=\alpha\left(1-2 \delta_{i j}\right)\left[\sum_{k=1}^{N}\left(E_{i} E_{j} E_{k}\right)^{1 / 6}\right] .
\end{aligned}
$$

Let $B_{i j}=\hat{q}_{i} \delta_{i j}$, then Eq. (13) becomes with the model assumptions

$$
\frac{d}{d t} E_{i}=\sum_{j=1}^{N}\left(A_{i j}+B_{i j}\right) E_{j}, \quad i=1, \ldots, N .
$$

Here, $A_{i j}$ describes the interactions due to internal interactions - equivalent to the spontaneous transition probability in spectroscopy. And $B_{i j}$ represents the energy absorbed from the environment - equivalent to the Einstein induced transition probability in atomic physics. The matrix $A_{i j}$ conserves energy while $B_{i j}$ does not.

In spectroscopy, there exists the same equation (17) for the population of energy shells in an atom. Here, $E_{i}$ corresponds to the population of electrons $n_{i}$ in the energy state $i$, the matrix $A_{i j}$ to the spontaneous decay from state $j$ to state $i$, and the matrix $B_{i j}$ quantifies external triggering. Energy conservation corresponds to preservation of the number of electrons. However, it should be noted that energy plays a different role in spectroscopy. In the Galerkin model, energy is a free parameter associated with mode $i$; in spectroscopy $i$ corresponds to a fixed energy level and $n_{i}$ takes over the role of the free parameter.

\section{Burgers equation - an illustrative example}

We illustrate the FTT model for a 1D partial differential equation, the Burgers equation. This equation represents a modified version of the 1D Navier-Stokes equation and mimics its dynamics in important aspects. First (Section 4.1), a Galerkin model of a modified Burgers equation is derived. Then (Section 4.2), the Galerkin solutions are compared against FTT predictions. In particular, 
truncated Burgers dynamics leading to an absolute equilibrium solution with equipartition of energy is considered. Thus, observations of Majda and Timofeyev [45] are reproduced and explained in the FTT framework.

\subsection{Galerkin model of a modified Burgers equation}

The Burgers equation

$$
\partial_{t} u+u \partial_{x} u=v \partial_{x x}^{2} u
$$

mimics the Navier-Stokes equation in the absolute derivative $D_{t} u:=\partial_{t} u+$ $u \partial_{x} u$ with quadratic nonlinearity and the dissipation term $v \partial_{x x}^{2} u$ with viscosity $v$. We add a convection term $U \partial_{x} u$ with constant velocity $U$ to include periodic traveling waves. This convection term arises from a change to a coordinate system moving with velocity $-U$. Moreover, a forcing term $g(x, t)$ shall balance the energy dissipation due to $v \partial_{x x}^{2} u$. The resulting equation reads

$$
\partial_{t} u+(U+u) \partial_{x} u=g(x, t)+v \partial_{x x}^{2} u .
$$

We consider $2 \pi$-periodic solutions of Eq. (18) in the Hilbert space of squareintegrable real functions $\mathcal{L}^{2}([0,2 \pi])$. The inner product of $u, v \in \mathcal{L}^{2}([0,2 \pi])$ is defined by

$$
(u, v)_{\Omega}:=\int_{0}^{2 \pi} d x u v .
$$

The trigonometric system $\left\{\Theta_{i}(x)\right\}_{i=0}^{\infty}$,

$$
\Theta_{i}(x):= \begin{cases}\frac{1}{\sqrt{2 \pi}} & i=0 \\ \frac{1}{\sqrt{\pi}} \sin \left(\frac{i+1}{2} x\right) & i>0 \quad \text { and } i \text { odd } \\ \frac{1}{\sqrt{\pi}} \cos \left(\frac{i}{2} x\right) & i>0 \quad \text { and } i \text { even }\end{cases}
$$

constitutes a complete, countable, orthonormal system of the Hilbert space. The time-dependent function $u(x, t)$ is approximated by the Galerkin expansion

$$
u^{[0 \ldots N]}(x, t):=\sum_{i=0}^{N} a_{i}(t) \Theta_{i}(x),
$$

where $a_{i}:=\left(u, \Theta_{i}\right)_{\Omega}$. 
The forcing of Eq. (18) is chosen to be $g(x, t):=\sigma\left[a_{1}(t) \Theta_{1}(x)+a_{2}(t) \Theta_{2}(x)\right]$. This term provides energy in the first spatial harmonics spanned by $\Theta_{1}, \Theta_{2}$ with growth rate $\sigma>0$. This linear dynamics mimics the role of the production term in the Navier-Stokes equation.

The resulting Galerkin system is obtained by a straightforward Galerkin projection of Eq. (18) on $\Theta_{i}, i=0, \ldots, N$. This projection yields a system of the form (6) [45]. The 0 -th equation reads $\dot{a}_{0}=0$. Without loss of generality, $a_{0} \equiv 0$ is assumed. The constant term vanishes, $c_{i}=0, i=1, \ldots, N$. The linear term has block diagonal structure with $2 \times 2$ matrix on the diagonal. And the quadratic term is energy preserving, i.e., fulfills Eq. (A 2) in Appendix A.

For $N=10$, the Galerkin system reads:

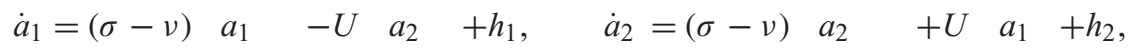

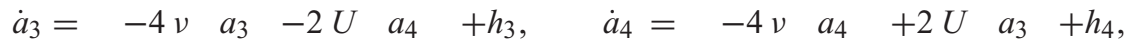

$$
\begin{aligned}
& \dot{a}_{5}=\begin{array}{lllllllllll}
-9 v & a_{5} & -3 U & a_{6} & +h_{5}, & \dot{a}_{6}= & -9 v & a_{6} & +3 U & a_{5} & +h_{6},
\end{array}
\end{aligned}
$$

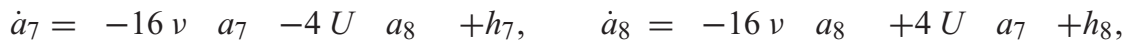

$$
\begin{aligned}
& \dot{a}_{9}=-25 v \quad a_{9} \quad-5 U \quad a_{10}+h_{9}, \quad \dot{a}_{10}=-25 v \quad a_{10}+5 U \quad a_{9}+h_{10},
\end{aligned}
$$

with the quadratic terms

$$
\begin{aligned}
& h_{1}=\left[-a_{1} a_{3}-a_{2} a_{4}-a_{3} a_{5}-a_{4} a_{6}-a_{5} a_{7}-a_{6} a_{8}-a_{7} a_{9}-a_{8} a_{10}\right] / \sqrt{\pi}, \\
& h_{2}=\quad\left[-a_{1} a_{4}+a_{2} a_{3}-a_{3} a_{6}+a_{4} a_{5}-a_{5} a_{8}+a_{6} a_{7}-a_{7} a_{10}+a_{8} a_{9}\right] / \sqrt{\pi}, \\
& h_{3}=2 \quad\left[+a_{1}^{2} / 2-a_{2}^{2} / 2-a_{1} a_{5}-a_{2} a_{6}-a_{3} a_{7}-a_{4} a_{8}-a_{5} a_{9}-a_{6} a_{10}\right] / \sqrt{\pi}, \\
& h_{4}=2 \quad\left[+a_{1} a_{2}-a_{1} a_{6}+a_{2} a_{5}-a_{3} a_{8}+a_{4} a_{7}-a_{5} a_{10}+a_{6} a_{9}\right] / \sqrt{\pi}, \\
& h_{5}=3 \quad\left[+a_{1} a_{3}-a_{1} a_{7}-a_{2} a_{4}-a_{2} a_{8}-a_{3} a_{9}-a_{4} a_{10}\right] / \sqrt{\pi}, \\
& h_{6}=3 \quad\left[+a_{1} a_{4}-a_{1} a_{8}+a_{2} a_{3}+a_{2} a_{7}-a_{3} a_{10}+a_{4} a_{9}\right] / \sqrt{\pi}, \\
& h_{7}=4 \quad\left[+a_{1} a_{5}-a_{1} a_{9}-a_{2} a_{6}-a_{2} a_{10}+a_{3}^{2} / 2-a_{4}^{2} / 2\right] / \sqrt{\pi}, \\
& h_{8}=4 \quad\left[+a_{1} a_{6}-a_{1} a_{10}+a_{2} a_{5}+a_{2} a_{9}+a_{3} a_{4}\right] / \sqrt{\pi}, \\
& h_{9}=5 \quad\left[+a_{1} a_{7}-a_{2} a_{8}+a_{3} a_{5}-a_{4} a_{6}\right] / \sqrt{\pi}, \\
& h_{10}=5 \quad\left[+a_{1} a_{8}+a_{2} a_{7}+a_{3} a_{6}+a_{4} a_{5}\right] / \sqrt{\pi} .
\end{aligned}
$$

The ten equations represent five quadratically coupled and harmonically related oscillators. The first oscillator for $i=1,2$ in Eq. (20) is amplified for supercritical conditions $\sigma>v$. The other oscillators are dampened in proportion to the square of their eigenfrequency.

\subsection{Galerkin solutions and FTT model}

For $v>\sigma$, the Galerkin solutions converges to the origin $a_{1}=a_{2}=\ldots=$ $a_{N}=0$. For the numerical analysis, we choose $\sigma=1 / 50, v=1 / 100$, $U=1$ and $N=10$ as reference condition. The resulting Galerkin solution 
Periodic solution

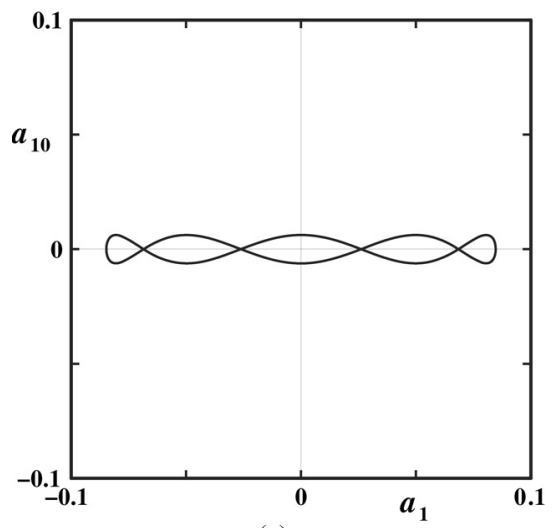

(a)
Absolute equilibrium solution

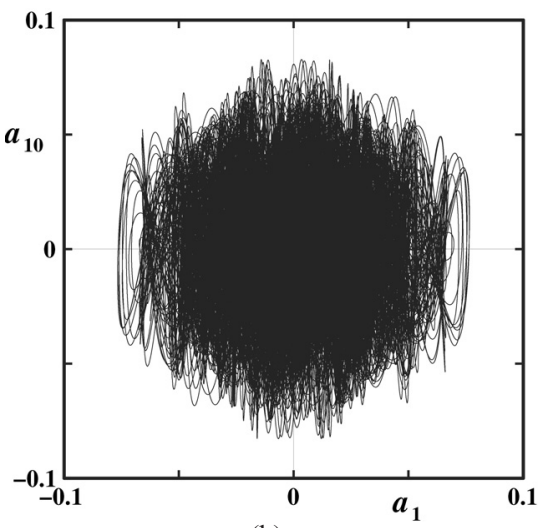

(b)

Figure 3 Phase portraits $a_{10}$ vs. $a_{1}$ of the Galerkin system (20). The Fourier coefficients $a_{1}(t)$ and $a_{10}(t)$ of the periodic solution (a) and the corresponding absolute equilibrium solution (b) are shown. The trajectory (b) is displayed for 5000 time units.

is periodic with circular frequency 1 , representing a self-sustained traveling wave with convection velocity $U=1$. Figure $3 a$ displays a phase portrait from the first and last Fourier coefficient. The portrait displays a Lissajous curve with frequency ratio 5 - corresponding to the eigenfrequency ratio of the first and last oscillator.

An interesting dynamics arises when production and dissipation of each oscillator is turned off, $v=\sigma=0$. In this case, the initial energy $E=$ $\left(a_{1}^{2}+\ldots a_{N}^{2}\right) / 2$ of the system is preserved. Each Fourier coefficient keeps a dominant frequency consistent with its eigenfrequency. Yet, the amplitudes vary irregularly and after a long time, a state with equipartition of energy is approximated,

$$
E_{i}=\overline{a_{i}^{2}} / 2 \approx E / N
$$

In fact, Majda \& Timofeyev [45] proof $E_{i} \rightarrow E / N$ as $N \rightarrow \infty$. These are called truncated Burgers [45] or absolute equilibrium solutions [22]. Figure $3 b$ shows a corresponding phase portrait. The resulting Galerkin expansion (19) does not approximate a solution of the Burgers equation. Yet, the Galerkin solution represent a well-studied limiting case of the Galerkin dynamics.

The modal energy distributions for the periodic and absolute equilibrium solutions are depicted in Figure 4. The latter is obtained by the first by setting $\sigma=v=0$ at some instant, i.e., both solutions have the some energy $E$. 
Periodic solution

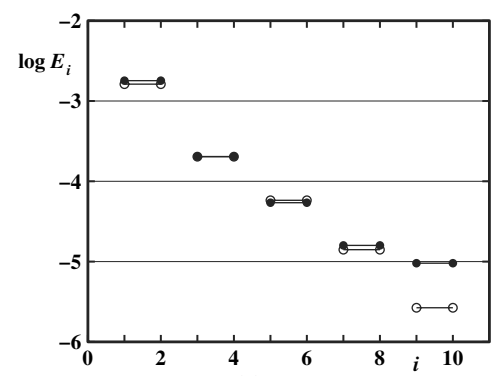

(a)

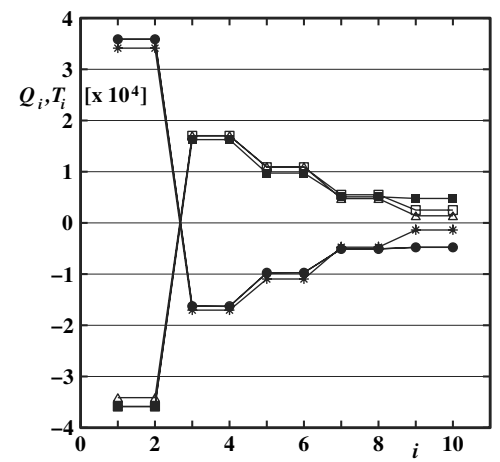

(c)
Absolute equilibrium solution

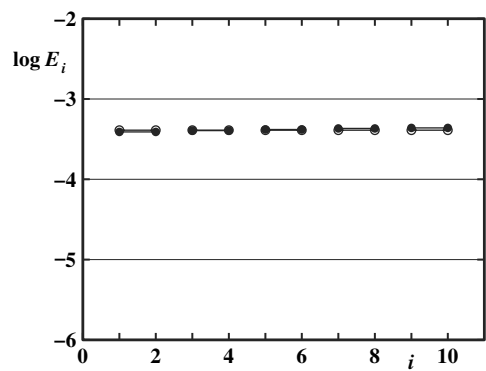

(b)

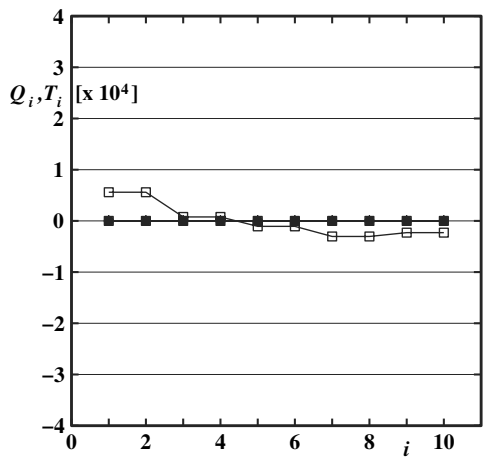

(d)

Figure 4 Energetics of the Galerkin system (20): (left: a,c) periodic solution, (right: b,d) corresponding absolute equilibrium solution. (top: a,b) Modal energy distributions from a numerical simulation $E_{i}(\bullet)$ and from the FTT model $\hat{E}_{i}(\circ)$. Pairs of trigonometric modes with the same wavenumber are connected by a line. (bottom: c,d) Energy flows re-scaled by $10^{4}$ : External energy flows $Q_{i}$ from the Galerkin solution $(\bullet)$, from the FTT analysis $\hat{Q}_{i}\left(E_{1}, \ldots, E_{10}\right)$ with the energy distribution $E_{i}$ of the Galerkin solution (०), and from the FTT model $\hat{Q}_{i}\left(\hat{E}_{1}, \ldots, \hat{E}_{10}\right)$ with estimated energy levels $\hat{E}_{i}(*)$. Internal energy flows: $T_{i}(\square), \hat{T}_{i}\left(E_{1}, \ldots, E_{10}\right)(\square)$, and $\hat{T}_{i}\left(\hat{E}_{1}, \ldots, \hat{E}_{10}\right)(\triangle)$. Note that some symbols are covered by the corresponding solid ones.

The Burgers equation fulfills all prerequisites for our finite-time thermodynamics formalism. It has a linear and quadratic term and the latter is energy preserving. The Galerkin-Reynolds equation (10) degenerates because the mean values $a_{i}=0, i=1, \ldots, N$, are not effected by the fluctuation. The modal energy equations (13) can be employed using the Galerkin system coefficients $c_{i}=0, l_{i j}$ and $q_{i j k}$ of Eq. (20). Numerical studies suggest that the equations have a single globally stable fixed point with attractive basin $\hat{E}_{i}>0$. The resulting FTT model predicts energy levels $\hat{E}_{i}$ which are in good agreement with converged ones of the Galerkin solutions $E_{i}$ (Figure $4 \mathrm{a}$ ). The deviation between $E_{i}$ and $\hat{E}_{i}$ for the periodic dynamics at $i=9,10$ is a truncation effect of the finite Galerkin expansion. Increasing the number of $N$ reduces this error. Moreover, the energy distribution converges rapidly with increasing $N$. 
In addition, the external and internal energy flows $Q_{i}$ and $T_{i}$ are well predicted by the FTT model (Figure 4c). Expectedly, the prediction is better for the computed energy distribution than for the FTT-predicted values. Again, a truncation effect is apparent in modes $i=9,10$.

The Galerkin solution of the truncated dynamics with $g \equiv 0, v=0$ displays a monotonously increasing modal energy $E_{i}$ with mode index $i$ (Figure $4 \mathrm{~b}$ ). The difference between the energy levels is $14 \%$, while the FTT model predicts exact equipartition for these conditions. The difference vanishes with increasing number of modes $N$. The modeled transfer term $\hat{T}_{i}$ with computed energy distribution $E_{i}$ predicts that modes with an energy above (below) the average value lose (gain) energy to restore equipartition of energy (See " $\square$ " in Figure 4d).

\section{Periodic vortex shedding - a "Lyman alpha" state of fluid motion}

The second example illustrates the proposed FTT formalism for 2D periodic vortex shedding behind a circular cylinder leading to the well-known von Kármán vortex street. Such flow is a paradigm of a self-excited, amplitudelimited flow oscillation discussed in all text books of fluid dynamics. The von Kármán vortex street might be compared with the Lyman-alpha series of the hydrogen atom in spectroscopy in terms of dynamic simplicity.

The incompressible flow is computed with a spectral element method [46,47] of high spatial resolution; further details for the employed simulation parameters can be found in [36]. In the following, all variables of that numerical Navier-Stokes (CFD) solution are nondimensionalized with the cylinder diameter $D$, the oncoming velocity $U$, and the density of the fluid $\rho$. Figure 5 shows the streamlines of the periodic wake at a Reynolds number $R e=100$ - well above the onset of vortex shedding at $R e_{\text {crit }}=47$ and well below the $3 \mathrm{D}$ transition around $R e_{\text {crit, } 2} \approx 180$ [48-50]. Note the waviness of the wake.

Following the pioneering work of Deane et al. [33], the flow is described by a ten-dimensional Galerkin model based on the proper orthogonal decomposition (POD) from the velocity field. This POD extracts the most energetic modes from velocity snapshots in the observation domain (see Figure 5). The POD modes can be grouped in pairs $(2 n-1,2 n)$ resolving the $n$-th harmonics and are displayed in [25]. The $2 n$-th mode looks like the $2 n-1$-th mode $(n=1,2,3, \ldots)$ shifted in streamwise direction. A similar behavior of the POD modes is observed in many other periodic shear flows [26,51]. 


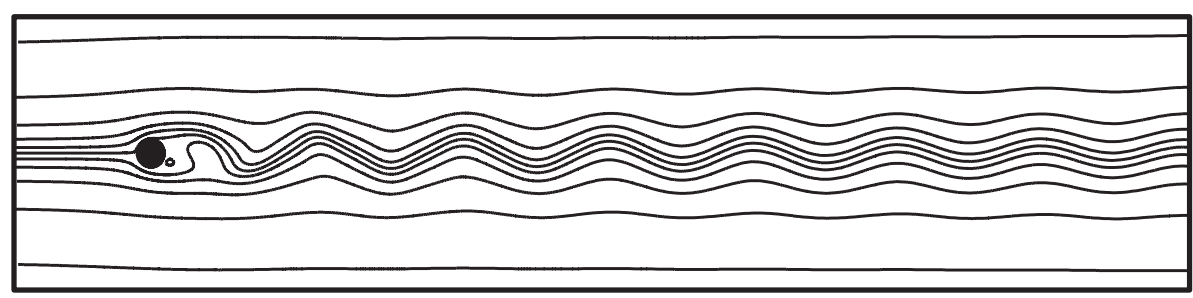

Figure 5 Cylinder wake at $R e=100$. The cylinder at $(x, y)=(0,0)$ is depicted by the solid circle. An instantaneous flow field is visualized by streamlines in the most active domain $x \in[-5,38]$, $y \in[-5,5]$. The computational region extends to $x \in[-20,38], y \in[-20,20]$.

By construction, the energy levels are sorted, $E_{1} \geq E_{2} \geq E_{3} \geq \ldots$. In addition, the energy level in the $n$-th pair of modes $(2 n-1,2 n)$ is nearly evenly distributed, i.e., $E_{2 n-1} \approx E_{2 n}$. Hence, the modal energy distribution $E_{i}$ forms a staircase pattern in Figure 6. While the second and third pair of modes are known to have similar energy, the energy of the following mode pairs decreases rapidly in geometric progression following an asymptotic theory [34]. Thus, the cumulative modal energy

$$
E^{[1 \ldots I]}:=E_{1}+E_{2}+\ldots+E_{I}
$$

converges rapidly against the total value $E$ as $I$ increases. The FTT-modeled energy distribution $\left\{\hat{E}_{i}\right\}_{i=1}^{N}$ resolves well the computed values extracted from the direct numerical simulation (see Figure 6). A truncation effect of the POD expansion is evident from a logarithmic representation of the energy levels like in the model for Burgers equation.

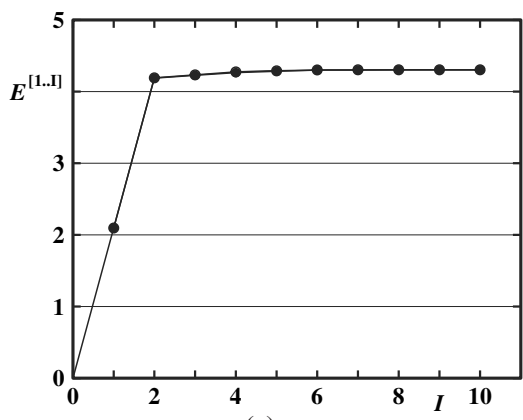

(a)

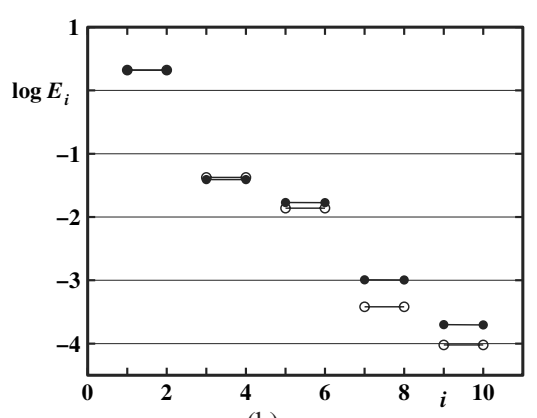

(b)

Figure 6 Energetics of the cylinder wake displayed in Figure 5. (a) Cumulative energy distribution $E^{[1 \ldots I]}=E_{1}+\ldots+E_{I}$ and (b) modal energy levels $E_{i}$ displayed on a logarithmic scale. The graphs display computed values $E_{i}(\bullet)$ and FTT-modeled pendants $\hat{E}_{i}(\circ)$. In (a), the difference between all $E_{i}$ and $\hat{E}_{i}, i=1, \ldots, 10$ is so small that the symbols overlap. In (b), pairs of modes with the same frequency but different phases are connected by a line. A truncation effect is apparent in the higher-order modes, like in the model of Burgers equation. 
The actual external interaction $Q_{i}$ is well approximated by the modeled value $\hat{Q}_{i}$. One reason is that the assumed diagonal structure of the correlation matrix (9) is a constitutive element of POD [5]. The computed and modeled transfer terms agree well also - explaining the good agreement of the corresponding energy distributions. Like in the model of Burgers equation, the first two POD modes provide the energy flow in the cascade that nurtures the remaining recipient modes.

The numerical results for periodic vortex shedding demonstrate that the FTT formalism provides a framework for predicting and interpreting the energy flow cascade. Also the onset of vortex shedding can be interpreted in this formalism (see Appendix B).

\section{Homogeneous shear turbulence - the "almost ionized state" of fluid motion}

As a last example, homogeneous shear turbulence is investigated assuming a periodic box. This flow is driven by a base flow with homogeneous shear. The temporal behavior gives rise to a broad-band frequency range - in contrast to the periodic cylinder wake. This large frequency range is reminiscent of spectra of almost ionized atoms. Despite dynamic complexity, the fluctuations are statistically homogeneous. That space-independent statistics makes homogeneous shear flow an El Dorado for theoretical studies and one of best investigated forms of turbulence $[22,52,53]$.

The flow is described in a Cartesian coordinate system. In that system, the base flow reads $\mathbf{u}_{0}=(S y, 0,0), S=d u / d y$ being the uniform shear rate (see Figure 7a). The fluctuation has wavelength $\lambda$ in $x$-, $y$-, and $z$-direction. The Reynolds number $R e=S \lambda^{2} / \nu$ is set to 1000 , where $v$ represents the kinematic viscosity. Following [9], the fluctuation is resolved by a Galerkin expansion with 1459 Stokes eigenmodes. Each mode is characterized by a wavenumber vector $\mathbf{k}=2 \pi \mathbf{n} / \lambda$, where $\mathbf{n}=\left(n_{1}, n_{2}, n_{3}\right)$ has integral components $n_{i}=$ $-4,-3, \ldots, 0,1, \ldots, 4$.

The ensemble of Stokes modes include 3D modes, 2D modes with one vanishing wavenumber component, and 1D flows resolving mean-flow deformations. In the following, the Stokes modes are numbered with a single index $i=1,2, \ldots, N$ and sorted with respect to the external energy flows $Q_{1} \geq Q_{2} \geq \ldots \geq Q_{N}$. This order separates donor modes with $Q_{i}>0$ and recipient modes with $Q_{i}<0$. The reduced-order Galerkin model (ROM) based on these modes resembles the large-scale irregular fluid motion (see Figure $7 b$ ) while it cannot resolve the turbulence cascade over orders of mag- 


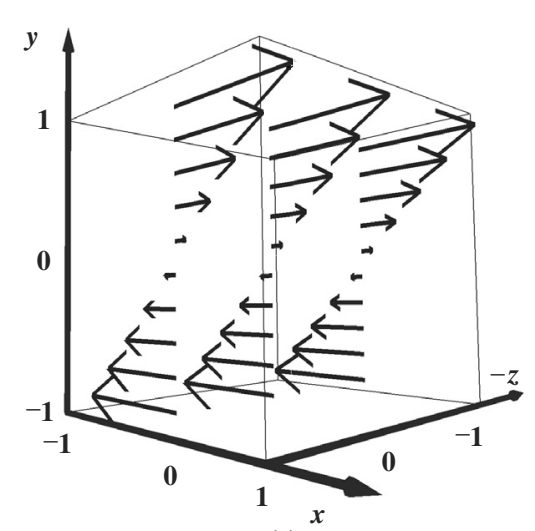

(a)

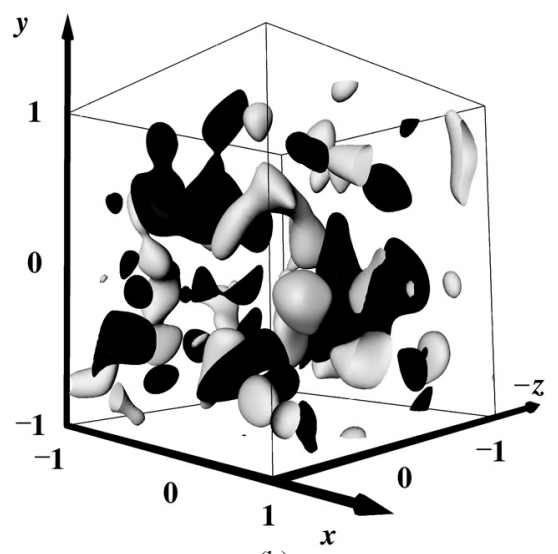

(b)

Figure 7 1459-dimensional Galerkin model of homogeneous shear turbulence at $R e=1000$. The figure displays the 1D mean flow (a) and a snapshot of the 3D fluctuation (b). In (b), the flow is represented by an iso-surface of a positive (bright) and negative $v$-component (dark).

nitudes. Such a resolution is even difficult for Navier-Stokes simulations on one the largest computers of this planet, the earth simulator [54].

The ROM serves its purpose for the proof-of-concept study of the proposed FTT formalism. We focus on the FTT analysis of the ROM solution for the internal interactions leading to $T_{i}$. The external pendant $Q_{i}$ is equal but opposite to $T_{i}$ due to convergence of the statistics (8). The model of this term $\hat{Q}_{i}$ has to be refined for an accurate FTT prediction of the energy level. This predictive capability will be elaborated in another forthcoming publication.

The time-averaged energetics of a converged ROM solution are displayed in Figure 8 . The cumulative modal energy $E^{[1 \ldots, I]}$ and transfer terms $T^{[1 \ldots I]}$ are shown. In contrast to modal values (see, e.g., Figs. 4 and 6b), such a distribution function filters out fluctuations. Evidently, the cumulative modal energy is not converged against an asymptotic value - thus indicating a truncation effect of the ROM. The transfer term has 432 energy-producing modes $\left(Q_{i}>0\right)$ that feed the energy flow cascade to the remaining dissipative modes $\left(Q_{i}<0\right)$. The large number of active modes is consistent with the observed broad-band frequency dynamics of the fluctuation. The FTT-modeled cumulative transfer term $\hat{T}^{[1 \ldots I]}$ follows well the ROM-computed values in the productive regime. In the dissipative regime, it deviates by up to $14 \%$. This deviation can partly be attributed to the severe spatial truncation of the Galerkin expansion. Energy flows to the neglected less energetic modes are not resolved in the framework of this ROM. For the given proof-of-concept ROM, the accuracy of the FTT formalism can be considered as satisfactory. 


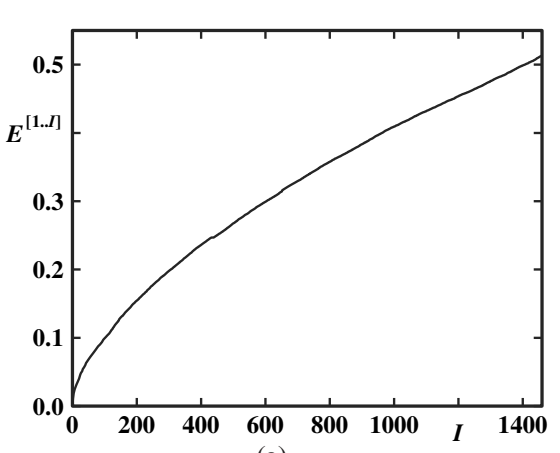

(a)

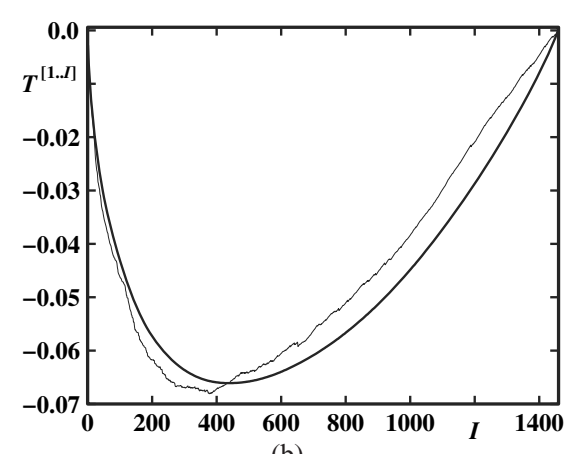

(b)

Figure 8 Energetics of the homogeneous shear turbulence displayed in Figure 7. (a) Cumulative energy distribution $E^{[1 . . I]}$; (b) computed and modeled cumulative transfer terms, $T^{[1 \ldots I]}$ (thick line) and $\hat{T}^{[1 \ldots I]}$ (thin line), respectively.

The ROM displays equipartition of energy (14) under the same conditions as for the Burgers equation (Section 4). The initial condition is a state of the converged Galerkin model. The external interactions are set to zero $Q_{i}=0$, $i=1, \ldots, N$ by enforcing the so-called truncated Euler dynamics. Here, the shear rate and the viscosity is set to 0 , thus inhibiting energy sources and sinks. Truncated Euler solutions in spectral methods also display equipartition of energy [22]. Setting only the shear rate to 0 , yields a well studied decay of turbulence (see Appendix C).

These ROM results for homogeneous turbulence corroborate that each mode may be considered as a thermodynamic degree of freedom. Without external interactions due to viscosity or mean flow, internal interactions lead to an equipartition of energy between these degrees of freedom. Internal interactions between modes thus serve the same balancing effect as collisions between molecules in gases. The nontrivial observed modal energy distribution can be directly linked to the external interactions $Q_{i}$. Large excess energies $Q_{i}$ lead to more energetic modes that feed the energy in the energy flow cascade to less energetic modes. The energy imbalance between the modes is thus a finite-time phenomenon associated with redistribution of modal energy. An infinitely fast redistribution would assure equipartition of energy between the modes, even for nonvanishing $Q_{i}$.

The conventional interpretation of the turbulence cascade is that energy flows from large scales to small scales. The FTT formalism indicates, however, that the primary mechanism is a cascade from energetic to less energetic modes. This may correspond to the conventional cascade since large-scale modes may be associated with large production and negligible dissipation $\left(Q_{i}>0\right)$, while fine-scale modes have negligible modal production and high dissipation, i.e., 
$Q_{i}<0$. However, FTT predicts an inverse cascade from small to large scales if the large scales are less energetic. These inverse cascades are indeed observed in wall turbulence as well as in free shear turbulence due to vortex pairing. Thus, FTT may comprise the normal and the inverse turbulence cascade in a unified framework.

\section{Conclusions}

This study proposes a finite-time thermodynamics formalism for unsteady incompressible fluid flows. That formalism builds upon an expansion of the flow field in terms of a mean flow $\mathbf{u}_{0}(\mathbf{x})$ and $N$ global modes $\mathbf{u}_{i}(\mathbf{x})$ with time-dependent amplitudes $a_{i}(t)$,

$$
\mathbf{u}(\mathbf{x}, t)=\mathbf{u}_{0}(\mathbf{x})+\sum_{i=1}^{N} a_{i}(t) \mathbf{u}_{i}(\mathbf{x}) .
$$

The global modes extend over the entire flow domain and are conceptionalized as waves. They may be Fourier modes, stability eigenmodes, or constitute a POD expansion. Each mode is characterized by a mean value of the Fourier coefficient $\overline{a_{i}}$ and an energy level $E_{i}=\overline{\left(a_{i}^{\prime}\right)^{2}} / 2$.

The statistical closure determines the first and second moments of the Fourier coefficients and thus the corresponding moments of the flow field. The closure builds on a Galerkin system with constant, linear, and quadratic terms,

$$
\dot{a}_{i}=c_{i}+\sum_{j=1}^{N} l_{i j} a_{j}+\sum_{j, k=1}^{N} q_{i j k} a_{j} a_{k}, \quad i=1, \ldots, N .
$$

The quadratic term alone leads to Hamiltonian (energy-preserving) dynamics. In particular, the term $q_{i j k}$ describes internal interactions between the modes. The linear term parameterized by $l_{i j}$ represents external interactions of the mode with the base flow $\mathbf{u}_{0}$ and the "molecular chaos" characterized by the kinematic viscosity $v$. The constant term $c_{i}$ is an important parameter in the mean-field equation. It vanishes if the steady solution is chosen as the base flow $\mathbf{u}_{0}$.

The proposed FTT formalism rests on the Galerkin-Reynolds equation, i.e., the averaged Galerkin system, and the modal energy flow balance [26]. Under statistically stationary conditions, the modal energy flow equation implies that for each mode the energy flows due to external interactions and internal inter- 
actions are in balance. From Eqs. (13) and (12), the balance equation reads

$$
\begin{aligned}
\dot{E}_{i}= & \underbrace{\hat{q}_{i} E_{i}}_{\substack{\text { external } \\
\text { interactions }}}+\underbrace{\sum_{j, k=1}^{N} \alpha \chi_{i j k}\left(E_{i} E_{j} E_{k}\right)^{1 / 2} \frac{\frac{1}{2}\left(E_{j}+E_{k}\right)-E_{i}}{E_{i}+E_{j}+E_{k}}}_{\begin{array}{c}
\text { internal } \\
\text { interactions }
\end{array}}=0, \\
& i=1, \ldots, N .
\end{aligned}
$$

The simplifications are axiomatically derived from known and postulated properties of the Galerkin system. The key assumption is that the external interaction term of one mode depends only on its energy and that each internal interaction term depends on the energies of the involved modes. These assumptions are inspired by energy shell models of turbulence theory.

Each mode is considered as a thermodynamic degree of freedom. The quadratic term of the Galerkin system alone leads to local thermal equilibrium (LTE), i.e., equipartition of energy. The linear term differentiates between donor and recipient modes of the energy flow cascade and promotes a complete order. The donor modes diverge and recipient modes decay assuming a frozen meanfield and neglecting internal interactions. Thus, linear and quadratic terms compete against each other for "complete order" and "complete disorder", respectively. The compromise is a partial LTE state that is communicated by the relevant time scales for energy exchange. If the welfare system acts much faster than the growth of individual fortunes, an egalitarian communist state is promoted. If the welfare system is slow and ineffective, a capitalist system with large individual fortunes is obtained. Formally, the $\alpha$-parameter in Eq. (21) allows the interpolation between complete order $(\alpha=0)$ and complete disorder $(\alpha=\infty)$. The constant term does not affect the energy flows directly, but via the interplay between modal energy distribution and mean values of the Fourier coefficients.

The FTT model has been applied to three different configurations: (a) the 1D Burgers equation, (b) the 2D periodic vortex shedding behind a circular cylinder, and (c) the 3D homogeneous shear turbulence. The underlying Navier-Stokes or Navier-Stokes-related equations are discretized with a Galerkin expansion allowing a very low-dimensional representation of the kinematics. In all cases, the internal energy exchange is well described by the FTT model. Moreover, that model predicts well the energy distribution for the $1 \mathrm{D}$ and $2 \mathrm{D}$ configurations. For the $3 \mathrm{D}$ flow, the large dimension of the problem complicates the solution of coupled nonlinear equations. A truncated dynamics gives rise to approximate local thermal equilibrium (LTE) in those cases where the quadratic term is exactly Hamiltonian. For the cylinder 
Table 1 Investigated configurations.

\begin{tabular}{llll}
\hline Aspect & Burgers equation & cylinder wake & $\begin{array}{l}\text { homogeneous } \\
\text { turbulence }\end{array}$ \\
\hline \hline $\begin{array}{l}\text { Evolution } \\
\text { equation }\end{array}$ & $\begin{array}{l}\text { 1D partial } \\
\text { differential equation }\end{array}$ & $\begin{array}{l}\text { 2D Navier-Stokes } \\
\text { equation }\end{array}$ & $\begin{array}{l}\text { 3D Navier-Stokes } \\
\text { equation }\end{array}$ \\
\hline $\begin{array}{l}\text { Boundary } \\
\text { conditions }\end{array}$ & periodic & $\begin{array}{l}\text { Dirichlet } \\
\text { and convective }\end{array}$ & periodic \\
\hline $\begin{array}{l}\text { Reynolds } \\
\text { number }\end{array}$ & $R e=1 / v=100$ & $R e=100$ & $R e=1000$ \\
\hline Modes & $\begin{array}{l}N=10 \\
\text { mathematical modes, }\end{array}$ & $\begin{array}{l}N=10 \\
\text { empirical (POD) modes } \\
\text { from numerical solution }\end{array}$ & $\begin{array}{l}\text { physical modes } \\
\text { (Stokes modes) }\end{array}$ \\
\hline $\begin{array}{l}\text { Optimality } \\
\text { of modes }\end{array}$ & completeness & data compression & linear dynamics \\
\hline Donor modes & 2 & 2 & 432 \\
\hline $\begin{array}{l}\text { Hamiltonian } \\
\text { quadratic term }\end{array}$ & exactly & approximately & exactly \\
\hline FTT analysis & $Q_{i}, T_{i}$ & $Q_{i}, T_{i}$ & $T_{i}$ \\
\hline FTT prediction & $E_{i}, \overline{a_{i}}$ & $E_{i}, \overline{a_{i}}$ & energy decay \\
\hline Truncated dynamics & approximate LTE & tendency to LTE & approximate LTE \\
\hline
\end{tabular}

wake, the truncated dynamics shows a strong tendency toward LTE. Table 1 summarizes the properties and FTT results of the investigated configurations. Forthcoming articles by the authors will address FTT models for mean-flow effects and algorithms for high-dimensional dynamics. Appendix D outlines a more general framework for this purpose.

An inspiring perspective of the FTT model is the possibility of a new path for control design. The nonlinear effect of a control law can be predicted over an infinite horizon. Thus, a nontrivial energy cascade manipulation may become possible, significantly generalizing control theory applications. The authors are actively pursuing this path for flow control applications.

The presented examples indicate that the FTT model works for a larger class of dynamical systems or Galerkin systems with Hamiltonian quadratic term. In all investigated cases, numerical experiments indicate that the attractor is robust and globally stable. Secondly, the low order of the modes induces a strong coupling between all modes. Good "mixing" in the state space can be expected to be beneficial to the performance of the proposed closure scheme. Certainly, we cannot expect the FTT formalism to overcome the sensitivity problem inhibiting all modeling approaches. If a small change in the dynamical system yields large changes in the solution, then small modeling errors have a similar effect. In particular, delicate resonance conditions will 
not adequately be predicted. Galerkin modes that do not yield similarly robust Galerkin systems might imply a poor performance of the FTT model. Largely, the class of systems appropriate for the proposed closure scheme will be determined by future studies.

An open question from a thermodynamics aspect is which functional nature tries to minimize in fluid flow systems. Can a version of the principle of minimum entropy production be derived on more formal grounds, e.g., the proposed modal balance equations? If yes, what are the side constraints and how are the thermodynamic fluxes and forces defined? If not, may the finite-time availability play a role in the new optimization functional, like in discussions of the Carnot engine $[55,56]$ ? In how far may thermodynamic optimization [57] be employed in flow control strategies, where actuation gives rise to additional energy flows $G_{i}$ into the $i$-th mode $[38,39]$ ? The thermodynamic heat flux analogy associated with Figure 2 suggests that at least some of these problems may be solvable within FTT. Moreover, FTT suggests a statistical mechanics for a Galerkin system attractor with probability distribution $p(\mathbf{a})$ employing the Shannon entropy $S=-\int d \mathbf{a} p(\mathbf{a}) \ln p(\mathbf{a})$ with suitable constraints.

In summary, it appears that the proposed FTT formalism offers a promising new path to the theoretical understanding of turbulence, to engineering computational models for this complicated flow phase, and to nonlinear flow control design. The authors are currently pursuing a turbulence model for the first and second statistical moments of engineering applications involving global stability eigenmodes and the FTT formalism. Future work should also aim at exploring the domain of applicability of our model.

\section{Acknowledgements}

We appreciate valuable stimulating discussions with Markus W. Abel, Laurent Cordier, Frank Curzon, Andreas Dillmann, Werner Ebeling, Helmut Eckelmann, Frank Jacobitz, William K. George, Hans-Christian Hege, Penelope Moffat, Dietmar Rempfer, Bernd Rummler, Peter Schmid, Jörn Sesterhenn, Frank Thiele, and Tino Weinkauf as well as the local TU Berlin team: Rudibert King, Oliver Lehmann, Mark Luchtenburg, Mark Pastoor, and Jon Scouten. This work was supported by the Deutsche Forschungsgemeinschaft (DFG) under grants NO258/1-1, NO258/2-3, SCHL 586/1-1, the Boye Ahlborn Turbulence Foundation, and the Bernd R. Noack Cybernetics Foundation. We acknowledge excellent working conditions and support of two DFG-funded Collaborative Research Centers: the Sfb 557 "Control of Complex Turbulent Shear Flow" at the Berlin University of Technology and the Sfb 609 "Elektromagnetische Strömungsbeeinflussung in Metallurgie, Kristallzüchtung und Elektrochemie" hosted at the Technical University Dresden. The work of 
Gilead Tadmor was partially supported by NSF grants 0410246 and 0524070 and the US AFOSR grants FA9550-05-1-0399 and FA9550-06-1-0373. The 3D flow visualization has been performed with Amira Software (Zuse Institute Berlin) [58]. We are grateful for outstanding computer and software support from Martin Franke and Lars Oergel. We are indebted to Steffi Stehr for carefully proof-reading the manuscript. Last but not least, we thank the referees whose encouraging and critical questions significantly contributed to the presented research results.

\section{Appendix A. Fick's law of triadic interactions}

Here, we describe the approximation of $T_{i j k}$ in Eq. (12). The nonlinearity is necessary for self-sustained unsteadiness and gives rise to the transfer term. The modal transfer term $T_{i}=\sum_{j, k=1}^{N} T_{i j k}$ comprises interactions between three modes involving the $i$-th mode, the so-called triadic interaction terms,

$$
T_{i j k}:=q_{i j k} \overline{a_{i}^{\prime} a_{j}^{\prime} a_{k}^{\prime}} \text {. }
$$

The main prerequisite is the energy-preserving property of the quadratic term in the Galerkin system, which implies

$$
q_{i j k}+q_{i k j}+q_{j i k}+q_{j k i}+q_{k i j}+q_{k j i}=0, \quad \forall i, j, k \in\{1, \ldots, N\} .
$$

This Hamiltonian property of the quadratic term can be derived from the Navier-Stokes equation for various boundary conditions [5,59], e.g., a periodic box, confined flow, or fluctuations in an ambient fluid. It is conserved by any orthonormal transformation of the expansion modes in the Galerkin approximation.

The key ansatz for modeling the triadic interaction term (A 1) is that the energy gain of the $i$-th mode in one triad $(i, j, k)$ depends only on the corresponding energy levels of the participating modes in that triad:

$$
\hat{T}_{i j k}=\hat{T}_{i j k}\left(E_{i}, E_{j}, E_{k}\right) .
$$

The transfer term may, however, depend on the Galerkin system coefficients involved in the triad, i.e., $q_{i j k}, q_{i k j}, \ldots, q_{k j i}$. It may be worthwhile to note that the spectral transfer terms in turbulence theory have been modeled in a similar spirit (see Appendix C).

The triadic interaction term fulfills a number of derivable or plausible properties:

P1) Homogeneity: Rescaling the mode amplitudes $a_{i}^{\prime} \mapsto \sqrt{\lambda} a_{i}^{\prime}$ with a positive parameter $\lambda$ yields $E_{i} \mapsto \lambda E_{i}$ and according to the triadic interaction 
term (A 1),

$$
\hat{T}_{i j k}\left(\lambda E_{i}, \lambda E_{j}, \lambda E_{k}\right)=\lambda^{3 / 2} \hat{T}_{i j k}\left(E_{i}, E_{j}, E_{k}\right) .
$$

P2) Zeros: The triadic interaction term (A 1) vanishes if $a_{i}^{\prime} \equiv 0\left(E_{i}=0\right)$ or $a_{j}^{\prime} \equiv 0\left(E_{j}=0\right)$ or $a_{k}^{\prime} \equiv 0\left(E_{k}=0\right)$. Hence,

$$
\hat{T}_{i j k}\left(0, E_{j}, E_{k}\right)=\hat{T}_{i j k}\left(E_{i}, 0, E_{k}\right)=\hat{T}_{i j k}\left(E_{i}, E_{j}, 0\right)=0 .
$$

P3) Energy conservation: The sum of all energy exchanges in one triad vanishes for a large class of boundary conditions [59]. In other words, the energy gained by one mode is lost by the other ones:

$$
\hat{T}_{i j k}+\hat{T}_{i k j}+\hat{T}_{j i k}+\hat{T}_{j k i}+\hat{T}_{k i j}+\hat{T}_{k j i}=0 .
$$

P4) Monotony: We assume that energy flows downhill, i.e. the least energetic mode of a triad wins and the most energetic one loses energy,

$$
\begin{aligned}
& E_{i}=\min \left\{E_{i}, E_{j}, E_{k}\right\} \Rightarrow \hat{T}_{i} \geq 0 ; \\
& E_{i}=\max \left\{E_{i}, E_{j}, E_{k}\right\} \Rightarrow \hat{T}_{i} \leq 0 .
\end{aligned}
$$

It should be noted that the downhill direction is only implied for the averaged quantities. Instantaneously, energy may, of course, flow uphill.

P5) Symmetry: Without loss of generality, the triadic transfer term can be assumed to be symmetric. Symmetrization does not affect the modal energy flow balance:

$$
\hat{T}_{i j k}=\hat{T}_{i k j} \text {. }
$$

P6) Realizability: The modeled transfer term should respect the upper and lower bounds arising from Eq. (A 1),

$$
\left|\hat{T}_{i j k}\right| \leq\left|q_{i j k}\right|\left|a_{i}^{\prime} a_{j}^{\prime} a_{k}^{\prime}\right|_{\max } \leq\left|q_{i j k}\right|\left|a_{i}^{\prime}\right|_{\max }\left|a_{j}^{\prime}\right|_{\max }\left|a_{k}^{\prime}\right|_{\max } .
$$

The word "realizability" is borrowed from similar constraints imposed on turbulence models for Reynolds' equation. Here, a positive trace of the modeled Reynolds tensor, i.e., positive turbulent kinetic energy, is required. We approximate $\left|a_{i}^{\prime}\right|_{\max }$ by $\sqrt{E_{i}}$ modulo a factor of order one. In this case, the strong realizability condition (A 4) is replaced by a softer version

$$
\left|\hat{T}_{i j k}\right| \lesssim\left|q_{i j k}\right| \sqrt{E_{i} E_{j} E_{k}},
$$

where the absolute value of the modeled term shall not exceed the limit on the right-hand side by a factor of larger order than unity. 
All properties $\mathrm{P} 1, \mathrm{P} 2, \mathrm{P} 5$, and $\mathrm{P} 6$ are derived or motivated from the definition of the triadic transfer term (A 1). Energy preservation P3 is synonymous with Eq. (A 2). The monotony property $\mathrm{P} 4$ is motivated by physical considerations. As a first illustrative case, we consider truncated Euler dynamics with $Q_{i}=0$, $i=1, \ldots, N$ displaying thermal equilibrium $E_{1}=E_{2}=\ldots=E_{N} . \mathrm{P} 4$ implies $\hat{T}_{i}=0, i=1, \ldots, N$, i.e., thermal equilibrium is indeed a solution of Eq. (13). As a second case, the turbulence cascade is considered. In the inertial range, K41 theory describes the decay of energy with the increase of the wavenumber $\kappa$,

$$
E(\kappa)=C \epsilon^{2 / 3} \kappa^{-5 / 3},
$$

where $C$ is the Kolmogorov constant and $\epsilon$ the dissipation per unit mass (see, e.g., [60]). According to that theory, modes at the wavenumber $\kappa$ receive energy from more energetic modes, at lower wavenumbers, and transfer it to less energetic ones, at higher wavenumbers. In other words, the assumed "downhill" flow of energy in P4 is qualitatively consistent with K41 theory describing an energy transfer from low to large wavenumbers. Finally, we consider the inverse cascade due to vortex merging. Here, energy flows from the energy-carrying coherent structures to emerging less energetic, larger scale structures. Hence, also the inverse cascade is qualitatively consistent with the assumed downhill energy flow in P4. In addition to these qualitative considerations, our numerical results for several 2D and 3D shear flows corroborate the tendency of least energetic modes to absorb energy from the most energetic ones in the considered triad, both for the normal and the inverse cascade.

We propose the following formulation of a triadic interaction term as a realization of $\mathrm{P} 1-\mathrm{P} 6$,

$$
\hat{T}_{i j k}=\alpha \chi_{i j k}\left(E_{i} E_{j} E_{k}\right)^{1 / 2}\left(\frac{\frac{1}{2}\left(E_{j}+E_{k}\right)-E_{i}}{E_{i}+E_{j}+E_{k}}\right),
$$

where the factor $\chi_{i j k}:=\frac{1}{6}\left(\left|q_{i j k}\right|+\left|q_{i k j}\right|+\left|q_{j i k}\right|+\left|q_{j k i}\right|+\left|q_{k i j}\right|+\left|q_{k j i}\right|\right)$ is determined by the Galerkin system and is totally symmetric $\chi_{i j k}=\chi_{i k j}=$ $\ldots=\chi_{k j i}$. We shall call this factor triadic interaction function of the Galerkin system, alluding to similar restrictive roles of the characteristic function in set theory and structure function in turbulence theory. The factor $\chi_{i j k}$ shall be set to 0 when the triple correlation $\overline{a_{i}^{\prime} a_{j}^{\prime} a_{k}^{\prime}}$ vanishes, e.g., when strictly periodic Fourier coefficients have incommensurable frequencies. Properties P1-P5 are easily validated. The realizability P6 is appreciated from

$$
-1 \leq \frac{\frac{1}{2}\left(E_{j}+E_{k}\right)-E_{i}}{E_{i}+E_{j}+E_{k}} \leq+1
$$


and assuming that the involved coefficients $q_{i j k}$ have the same order of magnitude.

The parameter $\alpha$ is determined so that the amount of energy spent by the donor modes is equivalent to the energy transferred by the donor modes to the recipient ones. Let $\mathcal{I}^{+}$be the index set of the donor modes with $\hat{Q}_{i}>0$, then $\alpha$ is determined from

$$
\sum_{i \in \mathcal{I}^{+}}\left[\hat{Q}_{i}+\hat{T}_{i}\right]=0 .
$$

Note that the modal energy flow balance is generally not fulfilled with the modeled terms, i.e., $\hat{Q}_{i}+\hat{T}_{i} \neq 0$ for all $i=1, \ldots, N$. This balance equation might, however, be satisfied by a mode-dependent parameter $\alpha_{i}, i=1, \ldots, N$, in the $\hat{T}_{i j k}$-ansatz.

We conjecture that Eq. (A 7) is the only nontrivial solution consistent with axioms P1-P6. The only degree of freedom is the design of the triadic interaction function. Another totally symmetric candidate is, for instance,

$$
\chi_{i j k}=\sqrt{\left(q_{i j k}^{2}+\ldots+q_{k i j}^{2}\right) / 6} .
$$

In fact, Eq. (A 7) has been distilled from a more general class of $\hat{T}_{i j k}$ that satisfies only properties P1-P5. Symmetry P5 and homogeneity P1 conditions suggest to employ powers of the product $E_{i} E_{j} E_{k}$ and the sum $E_{i}+E_{j}+E_{k}$ as normalization factor. Symmetry P5, energy preservation P3, and monotony $\mathrm{P} 4$ suggest $\left(E_{j}+E_{k}\right) / 2-E_{i}$ as redistribution factor. Homogeneity $\mathrm{P} 1$ restricts the permissible powers in the normalization factor resulting in

$$
\hat{T}_{i j k}=\alpha \chi_{i j k}\left(E_{i}+E_{j}+E_{k}\right)^{-\beta}\left(E_{i} E_{j} E_{k}\right)^{1 / 6+\beta / 3}\left(\frac{1}{2}\left(E_{j}+E_{k}\right)-E_{i}\right),
$$

where $\beta>0$ is a free parameter and $\chi_{i j k}$ is totally symmetric in the indices. Evidently, $\beta=1$ is the only choice consistent with P6 and this leads to Eq. (A 7).

An intriguing special case of Eq. (A 9) is obtained by setting $\chi_{i j k} \equiv 1$ for all $i, j, k \in\{1, \ldots, N\}$ and $\beta=0$,

$$
\hat{T}_{i j k}=\alpha\left(E_{i} E_{j} E_{k}\right)^{1 / 6}\left(\frac{1}{2}\left(E_{j}+E_{k}\right)-E_{i}\right) .
$$

This terms satisfies another property, namely 
P7 Universality: $\hat{T}_{i j k}\left(E_{i}, E_{j}, E_{k}\right)$ is assumed to be a universal function for all modal interactions independent of the Galerkin system,

$$
\hat{T}_{i j k}=F\left(E_{i}, E_{j}, E_{k}\right) .
$$

Note that universality (P6) and the realizability condition (P7) may not be compatible. For instance, $q_{i j k}=0$ implies $\hat{T}_{i j k}=0-$ consistent with the realizability condition. In contrast, mutually different energy levels $E_{i}, E_{j}$, and $E_{k}$ and a nontrivial universal solution imply $\hat{T}_{i j k} \neq 0$ in the ansatz (A 3 ).

For the numerical analysis, Eq. (A 7) is adopted. For thermodynamic interpretations, the simpler model (A 10) serves a better purpose. Generalization of these interpretations for the triadic interaction term (A 7) is straightforward. We propose the term Fick's law of triadic interaction for the $\hat{T}_{i j k}$ model; the reasons are outlined in Section 3.3.

\section{Appendix B. FTT model and linear stability analysis}

In Section 5, a FTT model of the 2D periodic vortex shedding at a supercritical Reynolds number has been proposed. Here, the onset of this vortex shedding is described. Thus, the interrelation between FTT and linear stability analysis is outlined.

At all Reynolds numbers $R e$, there exists a steady solution $\mathbf{u}_{s}$ of the NavierStokes equation. This solution becomes unstable at the critical Reynolds $R e_{\text {crit }}=47$. At $R e<R e_{\text {crit }}$, the steady flow is globally stable, i.e., all perturbations die out exponentially fast. At $R e>R e_{\text {crit }}$, a supercritical Hopf bifurcation gives rise to an oscillatory instability leading to periodic vortex shedding $[12,61,62]$.

This "phase transition" is described by a global stability analysis around the steady solution $\mathbf{u}_{s}$ [63]. At a given $R e$, the most amplified perturbation is of oscillatory nature and described by the real part of

$$
\mathbf{u}^{\prime}(\mathbf{x}, t)=e^{\lambda_{1} t} \mathbf{f}_{1}(\mathbf{x}) .
$$

The temporal behavior is characterized by the eigenvalue $\lambda_{1}=\sigma_{1}+l \omega_{1}$, where $\sigma_{1}$ represents the growth rate and $\omega_{1}$ the frequency. The spatial structure is resolved by the corresponding complex eigenmode $\mathbf{f}_{1}=\mathbf{u}_{1}+\imath \mathbf{u}_{2}$ with real part $\mathbf{u}_{1}$ and imaginary part $\mathbf{u}_{2}$. Figure 9 displays the steady solution and the most unstable eigenmode at a supercritical Reynolds number.

The dominant fluctuation thus lives in a two-dimensional subspace and can be described by a traditional Galerkin model based on the modes $\mathbf{u}_{1,2}$ after 


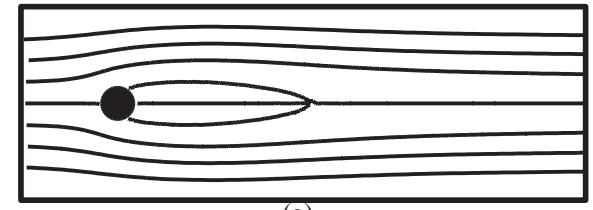

(a)

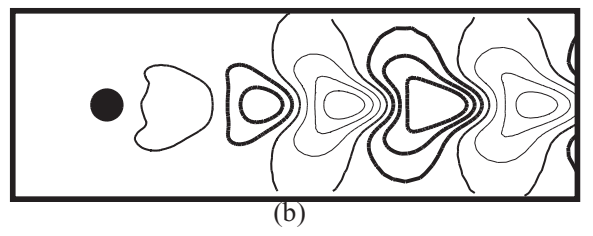

Figure 9 Steady solution (a) and most unstable eigenmode (b) of the cylinder wake at $R e=100$. The cylinder is represented by the solid circle. The flow fields are visualized by streamlines. Only the real part of the complex eigenmode is visualized. The imaginary part looks similar except for a quarter wave length stream-wise phase shift.

an orthonormalization. To simplify the following energy flow discussion, we assume that both modes have the same energy $E_{1}=E_{2}=E / 2$ and behave like the pair $\cos \omega_{1} t$ and $\sin \omega_{1} t$, i.e., are temporally uncorrelated. This assumption is observed to be a good approximation for instabilities of most free shear flows, including the cylinder wake.

The total energy exchange of the stability eigenmode with the external environment is given by

$$
\frac{d}{d t} E=Q=q E
$$

employing [26,36]. The transfer terms $T_{1,2}$ describe nonlinear interactions and are neglected in linear stability analysis. The external energy flow term $Q$ can be decomposed into the source $Q_{\text {prod }}$, due to interactions with the base flow $\mathbf{u}_{0}$, and the sink $Q_{\text {diss }}$, due to dissipation by viscous forces. The energy input from the base flow is given by $Q_{\text {prod }}=q_{\text {prod }} E$. This rate shall be called the (reduced) production, and the constant $q_{\text {prod }}$ the (reduced) production efficiency. This allows the definition of a production time scale $\tau_{\text {prod }}=1 / q_{\text {prod }}=\bar{E} / \overline{Q_{\text {prod }}}$ as the time needed for the production $\overline{Q_{\text {prod }}}$ to fill the energy level to $\bar{E}$. (The overbar indicates converged time-averaged values, as opposed to the instantaneous values from Eq. (B 1).) Similarly, the dissipation $Q_{\text {diss }}=q_{\text {diss }} E$ with dissipation efficiency $q_{\text {diss }}$ are introduced. The positive dissipation time scale

$$
\tau_{\text {diss }}=-1 / q_{\text {diss }}=-\bar{E} / \overline{Q_{\text {diss }}}
$$

represents the time needed for the dissipation $\overline{Q_{\text {diss }}}$ to absorb the energy $\bar{E}$. Summarizing, $q=1 / \tau_{\text {prod }}-1 / \tau_{\text {diss. }}$. From Eq. (B 1 ), the fluctuation is unstable at $q>0$. This implies $\tau_{\text {prod }}<\tau_{\text {diss }}$. In other words, a necessary and sufficient condition for linear instability is that a unit energy is produced quicker than it is dissipated. Similarly, stability implies $q<0$ and $\tau_{\text {prod }}>\tau_{\text {diss }}$. The critical Reynolds number is defined by $\tau_{\text {prod }}=\tau_{\text {diss }}$. This discussion suggests to 


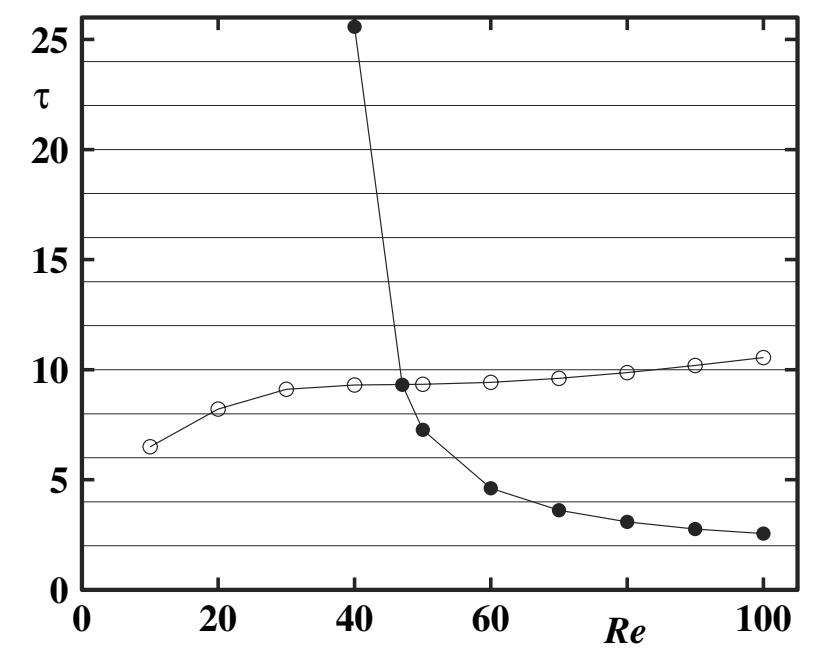

Figure 10 Onset of instability of the cylinder wake as transition-time phenomenon. The time scales for production $\tau_{\text {prod }}(\bullet)$ and dissipation $\tau_{\text {diss }}(\circ)$ are visualized for the most unstable eigenmode as a function of the Reynolds number.

introduce a new order parameter $R e^{\star}:=\tau_{\text {diss }} / \tau_{\text {prod }}$, for which we propose the name "onset Reynolds number." This parameter is proportional to Re and is unity at $R e_{\text {crit }}$.

Figure 10 displays the production and dissipation time scales in dependency of Reynolds numbers. As expected, $\tau_{\text {prod }}=\tau_{\text {diss }}$ at $R e=R e_{\text {crit }}=47$. Intriguingly, the dissipation time remains approximately constant at supercritical Reynolds numbers $R e>R e_{\text {crit }}$, while the production time diverges at $R e<R e_{\text {crit }}$. In other words, the most unstable modes become very inefficient in absorbing energy from the mean flow at $R e<R e_{\text {crit. }}$. Similar time-scale considerations for the onset of vortex shedding have been proposed in the vortex framework [64-66].

In the FTT formalism for $N$ modes, Eq. (13) implies that any energy distribution converges to the fixed point $E_{1}=E_{2}=\ldots=E_{N}=0$ if $q_{\max }:=$ $\max _{i \in\{1, \ldots, N\}}\left\{\hat{q}_{i}\right\}<0$. This can be proven by summing up the modal balances (13) to the total balance, exploiting energy preservation of the transfer term $T_{1}+T_{2}+\ldots+T_{N}=0$ and the estimate for the energy derivative

$$
\frac{d}{d t} E=q_{1} E_{1}+q_{2} E_{2}+\ldots+q_{N} E_{N} \leq q_{\max } E .
$$

In contrast, a sufficient and necessary criterion for a stable nontrivial energy distribution is the existence of one or more productive modes, i.e., $q_{\max }>0$. 


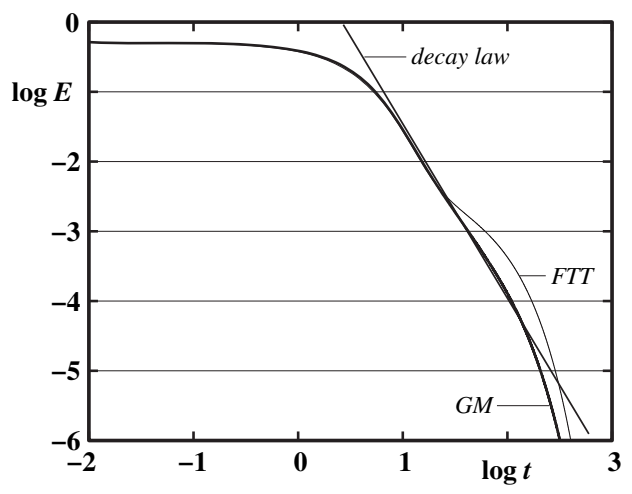

Figure 11 Decay of homogeneous shear turbulence. The shear rate is reduced to 0 at time $t=0$, thus removing the only source of energy in the cascade. The curves show the fluctuation energy of the Galerkin model of Section 6 (thick curve), the corresponding FTT model (thin curve), and a final decay law (C 1) (straight line) for the very final phase.

\section{Appendix C. FTT model and theory of homogeneous turbulence}

Here, we present a first consistency study between the FTT model and the theory of homogeneous turbulence. For this purpose, the 1459-dimensional Galerkin model of Section 6 is revisited. The Stokes modes resolve in each direction all wavenumbers up to $4 \times(2 \pi / \lambda), \lambda$ being the length of the periodic box.

We can corroborate that the Galerkin and FTT model are consistent with the decay law for the very final stage of turbulence [52],

$$
E \propto t^{-5 / 2} \text {. }
$$

The initial state of both models lies on the attractor of homogeneous shear turbulence discussed in Section 6. The decay starts at $t=0$ when the shear rate is set to 0 , i.e., when the production vanishes. Then, the total energy of the Galerkin model follows Eq. (C 1) well (see Figure 11). The kink near $t=20$ is related to an increase of the concentration of energy in the low-wavenumber modes, as the modes "feel" the limitations of the periodic box. This concentration is over-predicted by the FTT formalism. One reason is the frozen $\alpha$ parameter of $T_{i j k}$ for the shear turbulence state. Under statistically stationary conditions, $\alpha$ is well defined by an energy flow consistency between donor and recipient modes (A 8). Under the considered nonstationary decay conditions, no donor modes exists and no analogous consistency can be proposed.

Fully developed turbulence has also been studied extensively resting on the periodic box approximation $[52,67]$. The employed spectral methods fit into the 
traditional Galerkin framework, required by FTT models. Hence, in principle, the asymptotic properties of the proposed formalism can be compared against Kolmogorov's turbulence theory (see, e.g., [60]). In practice, the associated computational load inhibits meaningful numerical results: the reduced-order model above resolves only scales differing by a factor of at most 4 and involves already about 3 million triadic interactions. In general, the number of nonvanishing triadic interactions needed for the FTT model increases in proportion to $N^{2}$. Spectral methods are much more efficient employing an $N \log N$ algorithm [6,7]. However, a turbulence simulation with 4096 Fourier modes in each direction was performed already on one of the most powerful computers, the earth simulator [54]. Evidently, analytical approximations for $q_{i j k}$ and wavenumber interactions are necessary.

Analytical studies for periodic box turbulence are typically based on the spectral energy equation for decaying turbulence. Let $E(\kappa, t) d \kappa$ be the energy contained at time $t$ in the shell $\kappa<\|\boldsymbol{\kappa}\|<\kappa+d \kappa$, where $\boldsymbol{\kappa}$ represents the wavenumber vector, $\kappa$ its norm, and $d \kappa$ the width of the wavenumber shell. The time evolution is described by

$$
\partial_{t} E(\kappa, t)=-2 v \kappa^{2} E(\kappa, t)+T(\kappa, t),
$$

where $T(\kappa, t)$ is the transfer term representing all triadic interactions pumping energy in a sphere with unit width.

In any spectral Galerkin model, its dissipation term is well approximated by $D=2 \nu \kappa^{2} E(\kappa, t)$, because the coefficients of the viscous term $l_{i j}$ form a diagonal matrix. The challenge of turbulence modeling is an estimate of the transfer term. In the Galerkin model, the cumulative transfer term $\int_{0}^{\kappa} d \kappa^{\prime} T\left(\kappa^{\prime}, t\right)$ is a continuous approximation of all interactions where the first mode $i$ has wavenumber magnitudes $\left\|\boldsymbol{\kappa}_{i}\right\|<\kappa$ :

$$
\int_{0}^{\kappa} d \kappa^{\prime} T\left(\kappa^{\prime}, t\right)=\sum_{\substack{i, j, k \\\left\|\kappa_{i}\right\| \leq \kappa}} T_{i j k} .
$$

In the FTT model, the energy flow by a triadic interaction is given by $T_{i j k}=$ $T_{i j k}\left(E_{i}, E_{j}, E_{k}\right)$. In spectral closures, like Heisenberg's ansatz [68], $T(\kappa, t)$ is assumed to be a function of the whole energy distribution $\kappa \mapsto E(\kappa, t)$. In both closures, the energy distribution is the driving force of the transfer term. In the FTT model, a downhill flow toward thermal equilibrium is assumed, both for the normal and the inverse turbulence cascade. The spectral closure describes energy flow from low to large wavenumbers, in the inertial regime. Under these premises, neither the inverse cascade nor the absolute equilibrium ensemble is derivable. 
It should be noted the FTT and spectral closure have different benchmark equilibria. As a statistical physics ansatz, FTT predicts a vanishing transfer term $T \equiv 0$ for thermal equilibrium, i.e., $E \propto \kappa^{2}$ up to a maximum value of $\kappa_{\max }$. As asymptotic approximation, spectral closures, like Heisenberg's model, predict a vanishing transfer term for the inertial range obeying the $5 / 3$ law (A 6). Preliminary semi-analytical investigations of the authors addressing the 5/3 law in FTT framework are encouraging.

\section{Appendix D. Outline of a dynamic FTT model}

In some applications, the temporal evolution of coherent structures shall be explicitely resolved, e.g., shear flow fluctuations contributing to mixing and noise. To address this need, the FTT model is generalized for the triple decomposition into the base flow, time-resolved coherent structures and statistically modeled fluctuations [69]. A similar philosophy is adopted in a number of computational fluid dynamics approaches [70], e.g., URANS (unsteady Reynolds averaged Navier-Stokes) models and LES (large eddy simulation). The proposed generalization has already been successfully applied to the transient and post-transient cylinder wake.

The starting point is a Galerkin model with expansion (5) and dynamical system (6). We classify the modes $\mathbf{u}_{i}$ with $i \in \mathcal{I}=\{1, \ldots, N\}$ in three groups: dynamic, mean-field, and thermodynamic modes. The dynamic modes with $i \in \mathcal{I}_{\text {dyn }}$ may represent energetic coherent structures. Their evolution shall be resolved in time by a modified version of the ordinary differential equation (6):

$$
\dot{a}_{i}=c_{i}+\sum_{j \in \mathcal{I}_{\mathrm{dyn}} \cup{ }_{\mathcal{I}_{\mathrm{mf}}}} l_{i j} a_{j}+\sum_{j, k \in \mathcal{I}_{\mathrm{dyn}} \cup \mathcal{I}_{\mathrm{mf}}} q_{i j k} a_{j} a_{k}+\sigma_{i}\left(a_{i}-\overline{a_{i}}\right), \quad i \in \mathcal{I}_{\mathrm{dyn}} .
$$

Mean values $\overline{a_{i}}$ and associated fluctuation energies $E_{i}=\overline{\left(a_{i}^{\prime}\right)^{2}} / 2$ are estimated from the time history of $a_{i}(t)$. The last term is equivalent to a modal eddy viscosity ansatz $[15-17,71]$ and accounts for energy transfer to the thermodynamic modes. The parameter $\sigma_{i}$ is chosen to assure modal energy flow balance: $2 \sigma_{i} E_{i}=\sum_{\substack{j, k \in \mathcal{I} \\ j \vee k \in \mathcal{I}_{\mathrm{td}}}} \hat{T}_{i j k}$, where $\hat{T}_{i j k}$ is determined from Eq. (12).

The mean-field modes with $i \in \mathcal{I}_{\mathrm{mf}}$ may resolve slow base flow variations, e.g., by shift modes $[25,72,73]$. Their fluctuation is assumed negligible in the time window of interest $E_{i} \equiv 0$. The mean values $a_{i}=\overline{a_{i}}$ are described by the Galerkin-Reynolds equation (7) for $i \in \mathcal{I}_{\mathrm{mf}}$. The associated fluctuation energy vanishes, $E_{i}=0$, by assumption. 
The remaining thermodynamic modes with $i \in \mathcal{I}_{\text {td }}$ are statistically modeled as described in the current study, i.e., by Eqs. (10) and (13). Mean values $\overline{a_{i}}$ and modal energies $E_{i}$ of the other modes with $i \in \mathcal{I} / \mathcal{I}_{\text {td }}$ enter like the corresponding values of thermodynamic modes.

The dynamic generalization of the FTT, outlined above, includes the original FTT model $\left(\mathcal{I}=\mathcal{I}_{\text {td }}\right)$, the "pure" Galerkin system $\left(\mathcal{I}=\mathcal{I}_{\text {dyn }}\right)$, a variant of the slaving principle [74] $\left(\mathcal{I}_{\text {td }}=\varnothing\right)$, and myriad combination possibilities. The most important aspect of this generalization is that the coupling term between different sets of modes appears quite naturally. Moreover, the coupling term can be checked against the Galerkin-Reynolds equation and the modal energy balance equation as two important reference points.

The capability to resolve the time-evolution of critical modes in the dynamic FTT may significantly enhance its accuracy at low computational cost. Other currently pursued applications include dynamical least-order models from first principles and subgrid turbulence models.

\section{References}

[1] Lugt, H.J., Vortex Flow in Nature and Technology, 1st ed., Wiley-Interscience, New York, 1983.

[2] Visualization Society of Japan, Fantasy of Flow, 1st ed., IOS Press, Amsterdam, 1993.

[3] Berger, E., Wille, R., Periodic flow phenomena, Annu. Rev. Fluid Mech., 4 (1972), 313-340.

[4] Stuart, J.T., Nonlinear stability theory, Annu. Rev. Fluid Mech., 3 (1971), 347-370.

[5] Holmes, P., Lumley, J.L., Berkooz, G., Turbulence, Coherent Structures, Dynamical Systems and Symmetry, Cambridge University Press, Cambridge, 1998.

[6] Canuto, C., Hussaini, M.Y., Quarteroni, A., Spectral Methods in Fluid Dynamics, 3rd ed., Springer-Verlag, Berlin, 1993.

[7] Peyret, R., Spectral Methods for Incompressible Viscous Flow, 1st ed., SpringerVerlag, New York, 2002.

[8] Boberg, L., Brosa, U., Onset of turbulence in a pipe, Z. Naturforsch., 43a (1988), $697-726$.

[9] Rummler, B., Zur Lösung der instationären inkompressiblen Navier-Stokesschen Gleichungen in speziellen Gebieten (On the solution of the incompressible NavierStokes equations in some domains), Habilitation thesis, Fakultät für Mathematik, Otto-von-Guericke-Universität Magdeburg, 2000.

[10] Waleffe, F., On a self-sustaining process in shear flows, Phys. Fluids, 9 (1997), 883900.

[11] Moehlis, J., Faisst, H., Eckhardt, B., Periodic orbits and chaotic sets in a lowdimensional model for shear flows, SIAM J. Appl. Dyn. Syst., 4 (2005), 352-376.

[12] Landau, L.D., Lifshitz, E.M., Fluid Mechanics, Course of Theoretical Physics, Vol. 6, 2nd engl. ed., Pergamon Press, Oxford, 1987. 
[13] Boussinesq, J., Essai sur la théorie des eaux courantes, Mémoires présentés par divers savantsÆl' Académie des Sciences Paris, 23(1) (1877), 1-680 and 24(2) (1877), 1-60.

[14] Prandtl, L., Bericht über Untersuchungen zur ausgebildeten Turbulenz, Z. Angew. Math. Mech., 5 (1925), 136.

[15] Rempfer, D., Fasel, F.H., Dynamics of three-dimensional coherent structures in a flat-plate boundary-layer, J. Fluid Mech., 275 (1994), 257-283.

[16] Couplet, M., Sagaut, P., Basdevant, C., Intermodal energy transfers in a proper orthogonal decomposition - Galerkin representation of a turbulent separated flow, J. Fluid Mech., 491 (2003), 275-284.

[17] Tadmor, G., Noack, B.R., Dynamic estimation for reduced Galerkin models of fluid flows, 2004 American Control Conference, Paper WeM18.1 (2004), 0001-0006.

[18] Ebeling, W., Klimontovich, Y.L., Selforganization and Turbulence in Liquids, 1st ed., BSB B.G. Teubner Verlagsgesellschaft, Leipzig, 1984.

[19] Klimontovich, Y.L., Entropy and information of open systems, Physics Uspekhi, 42 (1999), 375-384.

[20] Loewen, S., Ahlborn, B., Filuk, A.B., Statistics of surface flow structures on decaying grid turbulence, Phys. Fluids, 29 (1986), 2388-2397.

[21] Chorin, A.J., Vorticity and Turbulence, 2nd ed., Springer-Verlag, Berlin, New York, 1997.

[22] Lesieur, M., Turbulence in Fluids, 2nd ed., Kluwer Academic Publishers, Dordrecht, Boston, London, 1993.

[23] Fletcher, C.A.J., Computational Galerkin Methods, 1st ed., Springer-Verlag, New York, 1984.

[24] Rempfer, D., Fasel, F.H., Evolution of three-dimensional coherent structures in a flat-plate boundary-layer, J. Fluid Mech., 260 (1994), 351-375.

[25] Noack, B.R., Afanasiev, K., Morzyński, M., Tadmor, G., Thiele, F., A hierarchy of low-dimensional models for the transient and post-transient cylinder wake, J. Fluid Mech., 497 (2003), 335-363.

[26] Noack, B.R., Papas, P., Monkewitz, P.A., The need for a pressure-term representation in empirical Galerkin models of incompressible shear flows, J. Fluid Mech., 523 (2005), 339-365.

[27] Curzon, F.L., Ahlborn, B., Efficiency of Carnot engine at maximum power output, Am. J. Phys., 43 (1975), 22-24.

[28] Andresen, B., Berry, R.S., Nitzan, A., Salamon, P., Thermodynamics in finite time. I. The step-Carnot cycle, Phys. Rev. A, 15 (1977), 2086-2093.

[29] Salamon, P., Andresen, B., Berry, R.S., Thermodynamics in finite time. II. Potentials for finite-time processes, Phys. Rev. A, 15 (1977), 2094-2102.

[30] Ahlborn, B., Curzon, F.L., Time scales for energy transfer, J. Non-Equilib. Thermodyn., 29 (2004), 301-312.

[31] Noack, B.R., Eckelmann, H., A low-dimensional Galerkin method for the threedimensional flow around a circular cylinder, Phys. Fluids, 6 (1994), 124-143.

[32] Ladyzhenskaya, O.A., The Mathematical Theory of Viscous Incompressible Flow, 1st ed., Gordon and Breach, New York, London, 1963.

[33] Deane, A.E., Kevrekidis, I.G., Karniadakis, G.E., Orszag, S.A., Low-dimensional models for complex geometry flows: application to grooved channels and circular cylinders, Phys. Fluids A, 3 (1991), 2337-2354. 
[34] Dušek, J., Le Gal, P., Fraunié, P., A numerical and theoretical study of the first Hopf bifurcation in a cylinder wake, J. Fluid Mech., 264 (1994), 59-80.

[35] Sirovich, L., Turbulence and the dynamics of coherent structures, Part II: symmetries and transformations, Quart. Appl. Math., XLV (1987), 573-582.

[36] Noack, B.R., Niederdimensionale Galerkin-Modelle für laminare und transitionelle freie Scherströmungen (Low-dimensional Galerkin models of laminar and transitional free shear flows), Habilitation thesis, Fakultät V - Verkehrs- und Maschinensysteme, Berlin University of Technology, 2006.

[37] Gerhard, J., Pastoor, M., King, R., Noack, B.R., Dillmann, A., Morzyński, M., Tadmor, G., Model-based control of vortex shedding using low-dimensional Galerkin models, 33rd AIAA Fluids Conference and Exhibit, AIAA Paper, 2003-4262 (2003).

[38] Noack, B.R., Tadmor, G., Morzyński, M., Low-dimensional models for feedback flow control. Part I: Empirical Galerkin models, 2nd AIAA Flow Control Conference, AIAA Paper 2004-2408 (2004).

[39] Tadmor, G., Noack, B.R., Morzyński, M., Siegel, S. Low-dimensional models for feedback flow control. Part II: Observer and controller design, 2nd AIAA Flow Control Conference, AIAA Paper 2004-2409 (2004).

[40] Samimy, M., Debiasi, M., Caraballo, E., Malone, J., Little, J., Özbay, H., Efe, M.Ö., Yan, X., Yuan, X., DeBonis, J., Myatt, J.H., Camphouse, R.C., Strategies for closedloop cavity flow control, 42nd Aerospace Sciences Meeting and Exhibit, AIAA Paper 2004-0576 (2004).

[41] Gad-el-Hak, M., Flow Control: Passive, Active and Reactive Flow Management, 1st ed., Cambridge University Press, Cambridge, 2000.

[42] King, R., Active Flow Control, 1st ed., Springer-Verlag, Berlin, 2007.

[43] King, R., Seibold, M., Lehmann, O., Noack, B.R., Morzyński, M., Tadmor, G., Nonlinear flow control based on a low dimensional model of fluid flow, in: Control and Observer Design for Nonlinear Finite and Infinite Dimensional Systems, LNCIS 322, Eds. T. Meurer, K. Graichen, E.D. Gilles, pp. 369-386, Springer-Verlag, Berlin, Heidelberg, 2005.

[44] Reif, F., Fundamentals of Statistical and Thermal Physics, 1st ed., McGraw Hill, New York, 1965.

[45] Majda, A.J., Timofeyev, I., Remarkable statistical behavior for truncated BurgersHopf dynamics, Proc. Natl. Acad. Sci. USA, 97 (2000), 12413-12417.

[46] Karniadakis, G.E., Sherwin, S.J., Spectral/HP Element Methods for Computational Fluid Dynamics, 2nd ed., Oxford University Press, Oxford, 2005.

[47] Mutschke, G., Gerbeth, G. Albrecht, T., Grundmann, R., Separation control of hydrofoils using Lorentz forces, Eur. J. Mech. B/Fluids, 25 (2006), 137-152.

[48] Zhang, H.-Q., Fey, U., Noack, B.R., König, M., Eckelmann, H., On the transition of the cylinder wake, Phys. Fluids, 7 (1995), 779-795.

[49] Barkley, D., Henderson, R.D., Three-dimensional Floquet stability analysis of the wake of a circular cylinder, J. Fluid Mech., 322 (1996), 215-241.

[50] Williamson, C.H.K., Mode A secondary instability in wake transition, Phys. Fluids, 8 (1996), 1680-1682.

[51] Ma, X., Karniadakis, G.E., A low-dimensional model for simulating threedimensional cylinder flow, J. Fluid Mech., 458 (2002), 181-190.

[52] Hinze, J.O., Turbulence, 2nd ed., McGraw Hill, New York, 1975. 
[53] McComb, D.W., The Physics of Fluid Turbulence, 1st ed., Clarendon Press, Oxford, 1991.

[54] Kaneda, Y., Ishihara, T., Yokokawa, M., Itakura, K., Uno, A., Energy dissipation rate and energy spectrum in high resolution direct numerical simulations of turbulence in a periodic box, Phys. Fluids, 15 (2003), L21-L24.

[55] Andresen, B., Finite-Time Thermodynamics, 1st ed., Physics Laboratory II, University of Copenhagen, Copenhagen, 1983.

[56] Chen, L., Wu, C., Sun, F., Finite time thermodynamic optimization or entropy generation minimization of energy systems, J. Non-Equilib. Thermodyn., 24 (1999), 327-359.

[57] Bejan, A., Entropy Generation Minimization. The Method of Thermodynamic Optimization of Finite-Size Systems and Finite-Time Processes, 1st ed., CRC Press, Boca Raton, New York, London, Tokyo, 1995.

[58] Stalling, D., Westerhoff, M., Hege, H.-C., Amira: A Highly Interactive System for Visual Data Analysis, in: The Visualization Handbook, Eds. C.D. Hansen, C.R. Johnson, pp. 749-767, Elsevier, Oxford, 2005.

[59] Kraichnan, R.H., Chen, S., Is there a statistical mechanics of turbulence?, Phys. D, 37 (1989), 160-172.

[60] Frisch, U., Turbulence, 1st ed., Cambridge University Press, Cambridge, 1995.

[61] Jackson, C.P., A finite-element study of the onset of vortex shedding in flow past variously shaped bodies, J. Fluid Mech., 182 (1987), 23-45.

[62] Zebib, A., Stability of viscous flow past a circular cylinder, J. Eng. Math., 21 (1987), $155-165$.

[63] Morzyński, M., Afanasiev, K., Thiele, F., Solution of the eigenvalue problems resulting from global non-parallel flow stability analysis, Comput. Meth. Appl. Mech. Eng., 169 (1999), 161-176.

[64] Ahlborn, B., Lefrançois, M., King, D., The clockwork of vortex shedding, Phys. Essays, 11 (1998), 144-154.

[65] Ahlborn, B., Seto, M.L., Noack, B.R., On drag, Strouhal number and vortex street structure, Fluid Dyn. Res., 30 (2002), 379-399.

[66] Bejan, A., Lorente, S., Constructural theory of generation of configuration in nature and engineering, J. Appl. Phys., 100 (2006), 041301.

[67] Batchelor, G.K., The Theory of Homogeneous Turbulence, reprint, Cambridge University Press, Cambridge, 1982.

[68] Heisenberg, W., Zur statistischen Theorie der Turbulenz (About the statistical theory of turbulence), Z. Phys., 124 (1948), 628-657.

[69] Reynolds, W.C., Hussain, A.K.M.F., The mechanics of an organized wave in turbulent shear flow. Part 3. Theoretical model and comparisons with experiments, J. Fluid Mech., 54 (1972), 263-288.

[70] Pope, S.B., Turbulent Flows, 1st ed., Cambridge University Press, Cambridge, 2000.

[71] Sirisup, S., Karniadakis, G.E., A spectral viscosity method for correcting the longterm behavior of POD models, J. Comp. Phys., 194 (2004), 92-116.

[72] Siegel, S., Cohen, K., McLaughlin, T., Feedback Control of a Circular Cylinder Wake in Experiment and Simulation, 33rd AIAA Fluids Conference and Exhibit, AIAA paper 2003-3571 (2003). 
[73] Bergmann, M., Cordier, L., Brancher, J.-P., Optimal rotary control of the cylinder wake using proper orthogonal decomposition reduced order model, Phys. Fluids, 17 (2005), 097101-097121.

[74] Haken, H., Synergetics, An Introduction. Nonequilibrium Phase Transitions and SelfOrganizations in Physics, Chemistry, and Biology. 3rd ed., Springer-Verlag, New York, 1983.

Paper received: 2006-12-29

Paper accepted: 2007-09-07 